\title{
Magnetic structures and reorientation transitions in noncentrosymmetric uniaxial antiferromagnets
}

\author{
A.N. Bogdanov, f U.K. Rößler, M. Wolf, and K.-H. Müller \\ Leibniz-Institut für Festkörper- und Werkstoffforschung Dresden \\ Postfach 270116 D-01171 Dresden, Germany
}

(Dated: June 4, 2018)

\begin{abstract}
A phenomenological theory of magnetic states in noncentrosymmetric tetragonal antiferromagnets is developed, which has to include homogeneous and inhomogeneous terms (Lifshitz-invariants) derived from Dzyaloshinskii-Moriya couplings. Magnetic properties of this class of antiferromagnets with low crystal symmetry are discussed in relation to its first known members, the recently detected compounds $\mathrm{Ba}_{2} \mathrm{CuGe}_{2} \mathrm{O}_{7}$ and $\mathrm{K}_{2} \mathrm{~V}_{3} \mathrm{O}_{8}$. Crystallographic symmetry and magnetic ordering in these systems allow the simultaneous occurrence of chiral inhomogeneous magnetic structures and weak ferromagnetism. New types of incommensurate magnetic structures are possible, namely, chiral helices with rotation of staggered magnetization and oscillations of the total magnetization. Fieldinduced reorientation transitions into modulated states have been studied and corresponding phase diagrams are constructed. Structures of magnetic defects (domain-walls and vortices) are discussed. In particular, vortices, i.e. localized non-singular line defects, are stabilized by the inhomogeneous Dzyaloshinskii-Moriya interactions in uniaxial noncentrosymmetric antiferromagnets.

PACS numbers: $75.10 .-b, 75.50 . E e, 75.30 . \mathrm{Kz}$
\end{abstract}

\section{INTRODUCTION}

In many magnetic crystals the magnetic properties are strongly influenced by the antisymmetric exchange (Dzyaloshinskii-Moriya) coupling which is generally described by a vector product formed by the magnetic moments $\mathbf{S}_{i}$ of two magnetic ions:

$$
w_{D}=\mathbf{D}_{i j} \cdot\left(\mathbf{S}_{i} \times \mathbf{S}_{j}\right)
$$

and the so-called Dzyaloshinskii vector $\mathbf{D}_{i j}$. 1 . $⿴$ B Based on phenomenological considerations, the interaction (11) was introduced by Dzyaloshinskii to explain the observation of a small net magnetization in a number of antiferfomagnets, a phenomenon called weak ferromagnetism 3 which is due to a slight deviation of the sublattice magnetizations from antiparallel arrangement. Extending Anderson's theory of superexchange Moriya later found a microscopic mechanism due to spin-orbit interactions that is responsible for the interactions (11). They arise in certain groups of magnetic crystals with low symmetry where the effects the couplings (11) do not cancel.2 During the following decades intensive theoretical and experimental studies on the Dzyaloshinskii-Moriya coupling (1) resulted in a deep insight into its microscopic origins and its manifestation in macroscopic properties of magnetic materials. 45 Now it is known that weak ferromagnetism must essentially be attributed to many types of complex magnetic structures. It influences appreciably the magnetic properties of several important classes of magnetic materials such as orthoferrites, manganites, some hightemperature superconducting cuprates, and others. 6.6

Another fundamental macroscopic manifestation of antisymmetric couplings (11) takes place in noncentrosymmetric magnetic crystals. Dzyaloshinskii showed that, in this case, the interaction (11) stabilizes long-periodic spatially modulated structures with fixed sense of rotation of the vectors $\mathbf{S}_{i}$. 0 Within a continuum approximation for magnetic properties, the interactions responsible for these modulations are expressed by inhomogeneous invariants. We will call these contributions to the (free) magnetic energy, involving first derivatives of magnetization or staggered magnetization with respect to spatial coordinates, inhomogeneous Dzyaloshinskii-Moriya interactions. They are linear with respect to the first spatial derivatives of the magnetization $\mathbf{M}$ of typed

$$
M_{i} \frac{\partial M_{j}}{\partial \eta}-M_{j} \frac{\partial M_{i}}{\partial \eta},
$$

where $M_{i}, M_{j}$ are components of magnetization vector(s) that arise in certain combinations in expressions (2) depending on crystal symmetry, and $\eta$ is a spatial coordinate. 7 Such antisymmetric mathematical forms first have been stydied in the theory of phase transitions by E. M. Lifshitz and are known as Lifshitz invariants. The first magnetic modulated chiral structures predicted ind were observed in the cubic noncentrosymmetric crystals MnSi and FeGe (space group $P 2{ }_{1} 3$ ). 10 During the following years modulated magnetic structures of this kind were discovered and investigated in several lasses of magnetic crystals lacking inversion center.11.12. 13 These chiral helical structures are essentially different from numerous other spatially modulated magnetic states in systems with competing exchange interactions (as, e.g., in rare earth metals) 11,44 The latter are characterized by rather short periods (usually including only few unit cells) and arbitrary rotation sense. On the contrary, chiral structures due to (11) or (2) have long period and a fixed sense of rotation. For example, in MnSi the periodicity lengths of the helix in zero magnetic field was found to be about $170 \AA$ (39 unit cells), and FeGe has an even larger period (700 $\AA$ or 149 unit cells). 9 The interactions of type (2) may also stabilize periodic structures mod- 
ulated in two-dimensions (vortex lattices) and localized axisymmetric inhomogeneous states. 15

Up to now both physical effects induced by the antisymmetric exchange coupling (1) — weakferromagnetism and chiral modulations - never have been observed simultaneously in one magnetic system. Moreover, in noncentrosymmetric magnetic crystals with chiral modulations that were known and described so far, the existence of weak ferromagnetism is excluded because of their symmetry.11 In this paper, we show that both phenomena can coexist in the recently discovered noncentrosymmetric tetragonal antiferromagnets $\mathrm{Ba}_{2} \mathrm{CuGe}_{2} \mathrm{O}_{7} 16.17$ and $\mathrm{K}_{2} \mathrm{~V}_{3} \mathrm{O}_{8} .18$ Due to the crystallographic and magnetic structures of these crystals, the Dzyaloshinskii-Moriya coupling (1) favours noncollinearity along one direction and spatial modulations along the others. Here we determine possible magnetic phases and study their evolution in applied magnetic fields. It turns out that the unique coexistence of weak ferromagnetism and chiral modulations enables the occurrence of new types of incommensurate structures and specific localized structures including so called magnetic vortices or skyrmions which are generally unstable in other classes of magnetic materials.

\section{THE MODEL}

\section{A. Phenomenological energy}

The tetragonal antiferromagnet $\mathrm{Ba}_{2} \mathrm{CuGe}_{2} \mathrm{O}+16,17$ (space group $P \overline{4} 2_{1} m$ ) helongs to the crystallographic class $D_{2 d}$ and $\mathrm{K}_{2} \mathrm{~V}_{3} \mathrm{O}_{8} \mathrm{~B}$ (space group $P 4 b m$ ) to $C_{4 v}$. The magnetic (free) energy within a continuum description consistent with the symmetry and the two-sublattice magnetic structure of these antiferromagnets can be derived by the standard approach to phenomenological theory.8 At temperatures sufficiently below the ordering temperature the vectors of sublattice magnetization $\mathbf{M}_{i}$ $(i=1,2)$ do not change their modulus. In this practically important case defined by neglecting the paraprocess, the vectors $\mathbf{M}_{i}$ have only orientational degrees of freedom and can be described by the unity vectors $\mathbf{m}_{i}=\mathbf{M}_{i} / M_{s}$, where $M_{s}=\left|\mathbf{M}_{1}\right|=\left|\mathbf{M}_{2}\right|$ is the sublattice saturation magnetization. For tetragonal antiferromagnetic crystals, the two-sublattice model described by the unity vectors $\mathbf{m}_{1}$ and $\mathbf{m}_{2}$ yields the magnetic energy in the following form:

$$
\begin{aligned}
W=\int\{ & \frac{\alpha}{2} \sum_{i=1}^{3}\left[\left(\frac{\partial \mathbf{m}_{1}}{\partial x_{i}}\right)^{2}+\left(\frac{\partial \mathbf{m}_{2}}{\partial x_{i}}\right)^{2}\right] \\
& +\alpha^{\prime} \sum_{i=1}^{3}\left(\frac{\partial \mathbf{m}_{1}}{\partial x_{i}} \frac{\partial \mathbf{m}_{2}}{\partial x_{i}}\right)+\frac{\lambda}{2} \mathbf{m}_{1} \cdot \mathbf{m}_{2} \\
& -\mathbf{h} \cdot\left(\mathbf{m}_{1}+\mathbf{m}_{2}\right) \\
& -\frac{\beta}{2}\left(m_{1 z}^{2}+m_{2 z}^{2}\right)-\beta^{\prime} m_{1 z} m_{2 z} \\
& \left.-d\left(m_{1 x} m_{2 y}-m_{2 x} m_{1 y}\right)+w_{D}\right\} d V .
\end{aligned}
$$

This includes inhomogeneous $\left(\alpha, \alpha^{\prime}\right)$ and homogeneous $(\lambda)$ parts of the exchange coupling and the interaction energy with the external field $\mathbf{h}$. The next two terms describe uniaxial second-order magnetocrystalline anisotropy with constants $\beta, \beta^{\prime}$, where the $z$-axis is taken along the tetragonal axis of the antiferromagnets. The homogeneous part of the Dzyaloshinskii-Moriya interaction with constant $d$ is responsible for weak ferromagnetism with small magnetic moments in the basal plane. Finally the energy contribution $w_{D}$ includes Lifshitz invariants of type (2). The functional form of $w_{D}$ depends on the crystal symmetry and will be specified later.

The next terms in a systematic expansion of the energy for a two-sublattice antiferromagnet are much weaker fourth-order terms of the magnetocrystalline anisotropy, including uniaxial parts with terms $m_{1 z}^{4}, m_{2 z}^{4}, m_{1 z}^{2} m_{2 z}^{2}$, and a magnetic anisotropy in the basal plane (XOY plane) composed of $x$ - and $y$-components of the vectors $\mathbf{m}_{i}$. The former are important in close vicinity to some reorientation transitions and the latter is responsible for small variations of magnetic structures when the magnetic field is rotated in the basal plane. These secondary effects are omitted in this contribution dedicated to the principal magnetic properties of the system. We also neglect the stray field contribution in the total energy (3) because, due to the antiparallel alignment of magnetic moments in antiferromagnets, stray fields are much weaker than in ferromagnetic crystals. They, however, play a crucial role in stabilizing multidomain structures in the vicinity of field-induced reorientation transitions. 19

The functional (3) includes all leading interactions in an uniaxial two-sublattice antiferromagnetic crystal. Here, we briefly list several special cases of the model (3) which describe important special classes of antiferromagnetic systems.

I.) $d=0, w_{D}=0$. Collinear antiferromagnets. The vast group of these antiferromagnetic materials ipcludes syeh well-studied species as $\mathrm{CuCl}_{2} \cdot 2 \mathrm{H}_{2} \mathrm{O} 20, \mathrm{MnF}_{2} 21, \mathrm{Cr}_{2} \mathrm{O}_{3} 22$, $\mathrm{GdAlO}_{3} 23$ (see for further references and review of their magnetic properties Refs.19 and24).

II.) $d \neq 0, w_{D}=0$. Antiferromagnets with weak ferromagnetism. In this case the energy (3) describes antiferromagnetic crystals with homogeneous DzyaloshinskiiMoriya interactions resulting in weak ferromagnetism 
or collinear antiferromagnets with "hidden" weak ferromagnetism. Among many others, this group includes $\mathrm{MnCO}_{3}, 3$ orthoferrites, 25 manganites, 26 and the most popular and well-studied weak-forromagnetic antiferro-

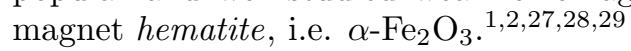

III.) $d=0, w_{D} \neq 0$. Chiral helimagnets. This case is realized in the cubic helimagnets discussed abpre and in other noncentrosymmetric magnetic systems.9.11 Usually interactions of type (2) stabilize modulated chiral structures in these materials11.

IV.) $d \neq 0, w_{D} \neq 0$. Chiral helimagnets with weak ferromagnetism. The model (3) represents previously unknown systems where both, homogeneous and inhomogeneous, Dzyaloshinskii-Moriya interactions are operational and not forbidden by any other additional symmetries. As will be shown in this paper, this unique coexistence of a mechanism for weak ferromagnetism and chiral coupling leads to specific modulated states with magnetization oscillations. Thus, in the following we will generally consider systems $d \neq 0$ and $w_{D} \neq 0$.

\section{B. Simplified model and basic equations}

It is convenient to use linear combinations of the sublattice magnetization $\mathbf{m}_{i}$, namely, the vector of total magnetization $\mathbf{m}=\left(\mathbf{m}_{1}+\mathbf{m}_{2}\right) / 2$ and the staggered magnetization (or vector of antiferromagnetic order) $\mathbf{l}=$ $\left(\mathbf{m}_{1}-\mathbf{m}_{2}\right) / 2$ as internal variables of the system. Because of $\left|\mathbf{m}_{i}\right|=1$ these vectors satisfy the constraints $\mathbf{m} \cdot \mathbf{l}=0, \mathbf{m}^{2}+\mathbf{l}^{2}=1$.

In most antiferromagnetic crystals the exchange coupling is much stronger than other internal interactions. Strong magnetic fields of order $\lambda$ destroy antiferromagnetic order and orientate the sublattice magnetizations $\mathbf{m}_{i}$ parallel to each other (a so called $s$ pin-flip transition into the "paramagnetic" phase with $|\mathbf{m}|=1, \mathbf{l}=0$ ). For most investigated antiferromagnetic systems these "exchange" fields are extremely large. Practically attainable values of magnetic fields usually only slightly distort the antiparallel arrangement inducing states with the total magnetization much smaller than unity. The hierarchy for the strength of interactions, $\lambda \gg d, \beta, \beta^{\prime}$, and the relations for the internal parameters, $m \ll 1$ and $l \approx 1$, permit to considerably simplify the energy (3) by excluding gradients of $\mathbf{m}$ and taking into account only the following terms (for details, see e.g.28)

$$
\begin{aligned}
\widetilde{W}=\int\{ & A \sum_{i=1}^{3}\left(\frac{\partial \mathbf{l}}{\partial x_{i}}\right)^{2}+\lambda \mathbf{m}^{2}-2 \mathbf{m} \cdot \mathbf{h} \\
& \left.+2 d\left(m_{x} l_{y}-m_{y} l_{x}\right)-B l_{z}^{2}+w_{D}\right\} d V,
\end{aligned}
$$

where $A=\alpha-\alpha^{\prime}$ and $B=\beta-\beta^{\prime}$. Functional forms of $w_{D}$ for all noncentrosymmetric crystallographic classes have been derived in Ref. 30. In particular, for the antiferromagnets under consideration the Lifshitz invariants quadratic in the components of $\mathbf{l}$ have the following form

$$
\text { class } D_{2 d}: w_{D}=D\left(l_{z} \frac{\partial l_{x}}{\partial y}-l_{x} \frac{\partial l_{z}}{\partial y}+l_{z} \frac{\partial l_{y}}{\partial x}-l_{y} \frac{\partial l_{z}}{\partial x}\right) \text {, }
$$

$\operatorname{class} C_{n v}: w_{D}=D\left(l_{z} \frac{\partial l_{x}}{\partial x}-l_{x} \frac{\partial l_{z}}{\partial x}+l_{z} \frac{\partial l_{y}}{\partial y}-l_{y} \frac{\partial l_{z}}{\partial y}\right)$.

The homogeneous part of the Dzyaloshinskii-Moriya interaction in (4) includes in-plane components of $\mathbf{m}$ and 1. They originate from the $z$-component of the vector product (11). Writing the Dzyaloshinskii vector in (14) as a sum of two parts proportional to $D$ and $d$, this contribution to (4) can be derived from the vector directed along the tetragonal axis $\mathbf{d}=(0,0, d)$. On the other hand, the Lifshitz invariants (5), (6) can be derived by an expansion for the in-plane components of the vector product (1) considering the contribution due to the vector $\mathbf{D}=(D, D, 0)$. The terminology in this field is not yet fixedly formulated. Here, following Ref. 31, we will call energy contributions given by Lifshitz invariants Dzyaloshinskii or chiral interactions to distinguish them from the homogeneous part of the Dzyaloshinskii-Moriya interaction.

Independent minimization of the energy (4) with respect to $\mathbf{m}$ leads to the following result

$$
\mathbf{m}=-[\mathbf{n} \times(\mathbf{d}+\mathbf{n} \times \mathbf{h})] / \lambda,
$$

where $\mathbf{n}=\mathbf{l} /|\mathbf{l}|$ is the unity vector parallel to the staggered magnetization vector. After substitution of (7) into the energy (4) one obtains the energy to leading approximation as a function of the vector $\mathbf{n}$

$$
\begin{aligned}
\widetilde{W}=\int\{ & A \sum_{i, j}\left(\frac{\partial n_{i}}{\partial x_{j}}\right)^{2} \\
& -\frac{1}{\lambda}\left[\left(h_{x}+d n_{y}\right)^{2}+\left(h_{y}-d n_{x}\right)^{2}-(\mathbf{h} \cdot \mathbf{n})^{2}\right] \\
& \left.-B n_{z}^{2}+w_{D}(\mathbf{n})\right\} d V
\end{aligned}
$$

where $w_{D}(\mathbf{n})$ is determined by (5) or (6). The energy (8) and (7) were derived from (3) by ignoring the paraprocess and assuming weak total magnetization, $|\mathbf{m}| \ll 1$, implying $|\mathbf{l}| \simeq 1$. Both assumptions are fulfilled in most realistic cases of interest. Thus, the energy (8) describing the orientation of the staggered magnetization can be considered as general phenomenological description for realistic uniaxial two-sublattice antiferromagnets. The functional (8) is related to so-called nonlinear $\sigma$-models which are basic subjects in the theory of solitons and which are intensively studied in mathematical and theoretical physics 32

The energy contributions (5) and (6) can result in states with modulations in the basal tetragonal plane, i.e. 
with a propagation vector in the $X O Y$-plane. Its actual direction is selected by some small in-plane anisotropy contributions that are neglected here (see discussion on expansion (3) above). On the other hand, there are no interactions in our systems violating homogeneity along the tetragonal $z$-axis. Hence, we infer that within the model (8) the most general solutions are inhomogeneous only in the basal plane but homogeneous along the $z$ axis. It is convenient to write the vector $\mathbf{n}(x, y)$ and the magnetic field $\mathbf{h}$ in spherical coordinates:

$$
\begin{aligned}
& \mathbf{n}=(\sin \theta \cos \psi, \sin \theta \sin \psi, \cos \theta), \\
& \mathbf{h}=(h \sin \zeta \cos \eta, h \sin \zeta \sin \eta, h \cos \zeta) .
\end{aligned}
$$

In these variables the total energy is given by

$$
\begin{aligned}
& \widetilde{W}=L_{z} \int\left\{A \sum_{i=1}^{2}\left[\left(\frac{\partial \theta}{\partial x_{i}}\right)^{2}+\sin ^{2} \theta\left(\frac{\partial \psi}{\partial x_{i}}\right)^{2}\right]\right. \\
& \left.+w_{D}+\tilde{w}\right\} d x d y,
\end{aligned}
$$

where the integration with respect to $z$ was performed for a system with linear size $L_{z}, x_{1}=x, x_{2}=y$. The Lifshitz invariants are given by

$$
\begin{aligned}
w_{D}=D[ & \sin \psi \frac{\partial \theta}{\partial x}+\cos \psi \frac{\partial \theta}{\partial y} \\
& \left.+\sin \theta \cos \theta\left(\cos \psi \frac{\partial \psi}{\partial x}-\sin \psi \frac{\partial \psi}{\partial y}\right)\right] \text { for } D_{2 d} \\
w_{D}=D[ & \cos \psi \frac{\partial \theta}{\partial x}+\sin \psi \frac{\partial \theta}{\partial y} \\
& \left.-\sin \theta \cos \theta\left(\sin \psi \frac{\partial \psi}{\partial x}-\cos \psi \frac{\partial \psi}{\partial y}\right)\right] \text { for } C_{n v},
\end{aligned}
$$

and the energy term $\tilde{w}$ does not depend on spatial derivatives:

$$
\begin{aligned}
\lambda \tilde{w}= & -\left(\lambda B-d^{2}-h^{2} \cos ^{2} \zeta\right) \cos ^{2} \theta \\
& -\left(h^{2} \sin ^{2} \zeta+d^{2}\right)+h^{2} \sin ^{2} \zeta \cos ^{2}(\psi-\eta) \sin ^{2} \theta \\
& -2 d h \sin \zeta \sin \theta \sin (\psi-\eta) \\
& +h^{2} \sin \zeta \cos \zeta \sin 2 \theta \cos (\psi-\eta) .
\end{aligned}
$$

The functional (10) with (13) provides the basic expression for the total energy of uniaxial two-sublattice antiferromagnets belonging to crystallographic classes without inversion symmetry. By inserting the appropriate Lifshitz invariant from (11) or (12) for $w_{D}$, the functional describes the magnetic energy of the two tetragonal crystals of interest here.

\section{THE PHASE DIAGRAM OF EQUILIBRIUM SOLUTIONS}

The equilibrium distributions of $\theta(x, y)$ and $\psi(x, y)$ are determined by solving a set of equations minimizing the energy (10). Depending on the values of the phenomenological constants in the energy (10) and the components of magnetic fields different spatially homogeneous and modulated phases can be realized in the system. Due to isotropy of the model in the basal plane only the component of magnetic field along the tetragonal axis $\left(h_{z}\right)$ and the value of its projection onto the basal plane $h_{\perp}$ are of importance. A reduction of the number of control parameters is obtained by rescaling the spatial variables and the energy. We use the following units for lengths, magnetic field, and the strength $D$ of the inhomogeneous Dzyaloshinskii-Moriya interaction

$$
\begin{aligned}
x_{0}=\sqrt{A \lambda /|K|}, & h_{0} & =\sqrt{|K|}, \\
D_{0}=\frac{4}{\pi} \sqrt{A|K| / \lambda}, & K & =\lambda B-d^{2}
\end{aligned}
$$

introducing an effective anisotropy constant $K$ acting on the staggered magnetization that is comparable to a constant of uniaxial anisotropy in ferromagnets. In centrosymmetric antiferromagnets $(D=0)$ and zero external field, the collinear state with staggered magnetization $\mathbf{l}$ along the tetragonal axis and $m=0$ is the ground state for $K>0$ (easy-axis system); for $K<0$ the vectors $\mathbf{l}$ and a nonzero $\mathbf{m}$ lie in the in the basal plane (weak ferromagnetic states). The characteristic length $x_{0}$ is of the order of the effective size of an isolated domain wall between the homogeneous states at zero field. In uniaxial ferromagnetic materials the corresponding expression for an intrinsic length is known as exchange or Bloch length. 33 The characteristic field $h_{0}$ is the so-called spinflop field. Finally, the parameter $D_{0}$ equals the lowest value of the (Dzyaloshinskii) constant $D$ that stabilizes modulated states at zero field (see below). In these reduced units (14), the energy (10) includes as independent parameters the rescaled constants $K$ involving $d$ and $D$ as well as the two components of the applied field $\left(h_{z}\right.$, $\left.h_{\perp}\right)$. Thus, these parameters span a four-dimensional phase-space for the solutions.

Before giving the detailed analysis, let us point out some general features of the possible magnetic configurations in this system. The equilibrium magnetic structures are governed by two opposing tendencies. The rotation of the staggered vector $\mathbf{l}$ with propagation vectors in the basal plane and an appropriate sense of rotation leads to negative values of the invariants (11), (12). An unlimited reduction of the pitch for this winding of the staggered magnetizations would lead to infinitely negative values of this Dzyaloshinskii energy. This is counter-acted by the inhomogeneous part of the exchange energy in (10) providing the "stiffness" of the magnetic structure. In isotropic systems, i.e. $\tilde{w}=0$ for expression (13), the ratio of these competing energy contributions yields the optimal period for the spiral, which is of the order of $A / D$. Such chiral modulations with uniform rotation are observed in low-anisotropy systems as cubic helimagnets 12 or in hexagonal chiral magets with in-plane rotation of the magnetization vectors. 13 The uniform rotation of 1 in spirals is disturbed by anisotropic interactions and/or 
by application of a magnetic field, i.e. the energy terms included in (13). These interaction terms result in preferred directions for the staggered vector $\mathbf{l}$ corresponding to the minima of the energy density (13). Hence, they distort the chiral modulations and may even suppress them by forcing the staggered magnetization to point fixedly into "easy" directions. Thus, chiral modulations may occur only beyond a certain threshold: the interactions (5) or (6) must be strong enough to overcome the anisotropic energy contributions suppressing modulated states. Below this threshold the system takes on the homogeneous states which are determined by minimization of the energy (10) with $D=A=0$. In the following subsections we will demonstrate this competition between homogeneous and inhomogeneous states for our model in detail.

Another important property of the system is related to the role played by the homogeneous DzyaloshinskiiMoriya interaction: in-plane components of the staggered vector induce corresponding non-zero components of the total magnetization vector $\mathbf{m}$ (weak-ferromagnetic moments) according to eq. (7). Thus, collinear antiferromagnetic states, $\mathbf{m}=0$, exist only when the staggered vector $\mathbf{l}$ is parallel to the tetragonal axis. All magnetic structures with a vector $\mathbf{l}$ deviating from this direction perforce have a locally nonzero magnetization $\mathbf{m}$. In zero field, these magnetic moments are in the basal plane. Helix-states of $\mathbf{l}$ are accompanied by magnetization components oscillating in sign and with the same period as the antiferromagnetic modulations. This peculiar mechanism leading to modulated antiferromagnetism and a related magnetization $\mathbf{m}$ may become operational and important, even if the ground-state is not modulated, for the more general case $\mathbf{h} \neq 0$ (see below).

In the general case the four dimensional phase diagram $\left(d, D, h_{z}, h_{\perp}\right)$ includes regions with different modulated and homogeneous states separated by "hypersurfaces" corresponding to different phase transitions. As remarked above, there are numerous experimental and theoretical results on magnetic properties of centrosymmetric antiferromagnets (model 4 with $w_{D}=0$ ) describing weak ferromagnetism.25 2. 20 The phase space of the control parameters in this case $\left(d, h_{z}, h_{\perp}\right)$ was found to have a very complex topology and, depending on the orientations and the relative strengths of the vectors $\mathbf{h}$ and $\mathbf{d}$, anumber of non-trivial transitions occur in these systems 28 This phase diagram can be considered as a "cross-section" given by the three-dimensional "hyperplane" $D=0$ through the general $\left(d, D, h_{z}, h_{\perp}\right)$-space investigated here. On the other side in chiral antiferromagnets described by the case $d=0$ for our model, both the spin arrangements in a spiral and the corresponding propagation directions are found to be very sensitive to the orientation and strength of the applied field. 34 In the general case of nonzero values of the constants $d$ and $D$, there is an even wider variety of homogeneous and inhomogeneous solutions characterized by complex noncollinear magnetic structures and variable directions of propagation vectors. Clearly, the full set of the homogeneous and inhomogeneous states corresponding to the energy (10) together with (11) to (13) are of general interest and well-worth of further investigations. Here, we restrict ourselves to the approximation valid for antiferromagnetic systems with small magnetic anisotropy and their specific hierarchy of the interactions. The physically expected relation $d \gg B$ turned out to be valid in all known systems with weak ferromagnetism and is based on the common relativistic origin of both magnetic energy contributions in these (low-anisotropy) systems. It is specifically valid for systems in which magnetism is due to d-electrons. In these systems, the Dzyaloshinskii-Moriya interactions generally overcome the magnetocrystalline anisotropy. This is expected to apply also in noncentrosymmtric antiferromagnets with d-electron magnetism for which the values for $d$ are still unknown. This allows to narrow considerably the range of physically meaningful control parameters in our model.

As already discussed above, the vector $\mathbf{d}$ induces a total magnetization in the basal plane which tends to orientate itself parallel to the in-plane components of an applied field $h_{\perp}$. Correspondingly, the staggered magnetization is rotated into the plane perpendicular the $h_{\perp}$ direction. The stronger the values of $d, h_{\perp}$ and of the in-plane components of $\mathbf{l}$ the stronger is this effect. We may assume that the deviation of the staggered magnetization from this plane perpendicular to the $h_{\perp}$ direction $\varepsilon=|\psi-\eta-\pi / 2|$ is small. By optimizing the energy (13) with respect to $\varepsilon$ one obtains

$$
\varepsilon=|\psi-\eta-\pi / 2|=\left|\frac{h \cos \zeta \cos \theta}{d+h \sin \zeta \sin \theta}\right|
$$

providing the consistency criterion for our assumption $\varepsilon \ll 1$. This approximation is valid always for $d \gg h$ and generally in a broad range of the orientations for the vectors $\mathbf{h}$ and $\mathbf{l}$. This includes almost all physically interesting cases. In the following analysis, we assume as central approximation that the staggered magnetization is always restricted to the plane perpendicular to $h_{\perp}$, i.e. $\varepsilon=0$. By substituting $\psi-\eta=\pi / 2$ the energy density (13) can be simplified and reduced to the following form using the scaled quantities (14)

$$
\begin{gathered}
\tilde{w}=\frac{|K|}{\lambda} \Phi(\theta) \quad \text { with } \\
\Phi(\theta)=\operatorname{sgn} K\left(1-\frac{h^{2}}{K} \cos ^{2} \zeta\right)(\sin \theta-\nu)^{2} \\
\nu=\frac{d h \sin \zeta}{K-h^{2} \cos ^{2} \zeta}
\end{gathered}
$$

(Here, we drop constant terms in $\tilde{w}$, i.e. those independent of $\theta$.) In the following subsections, we investigate spatially homogeneous phases, helical phases, and their respective stability limits with the approximation (16). 

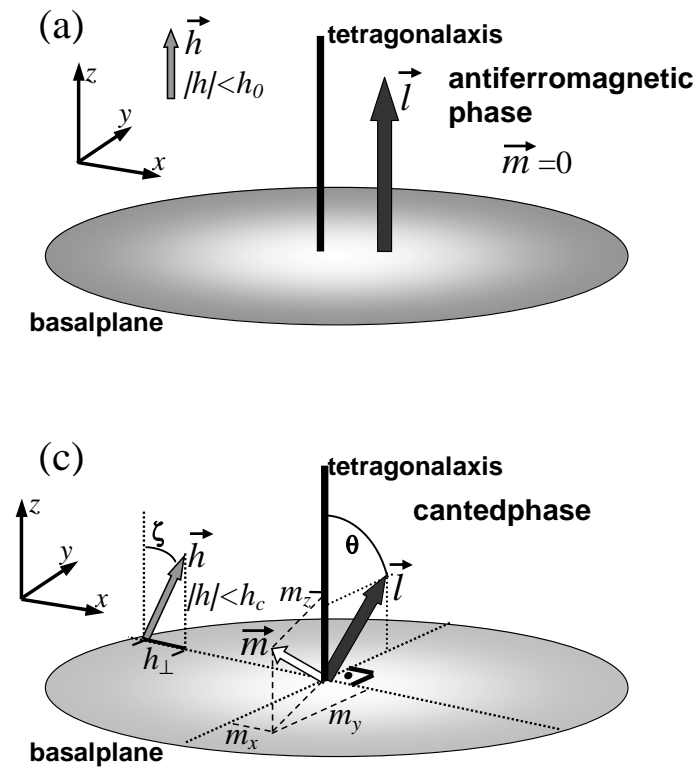
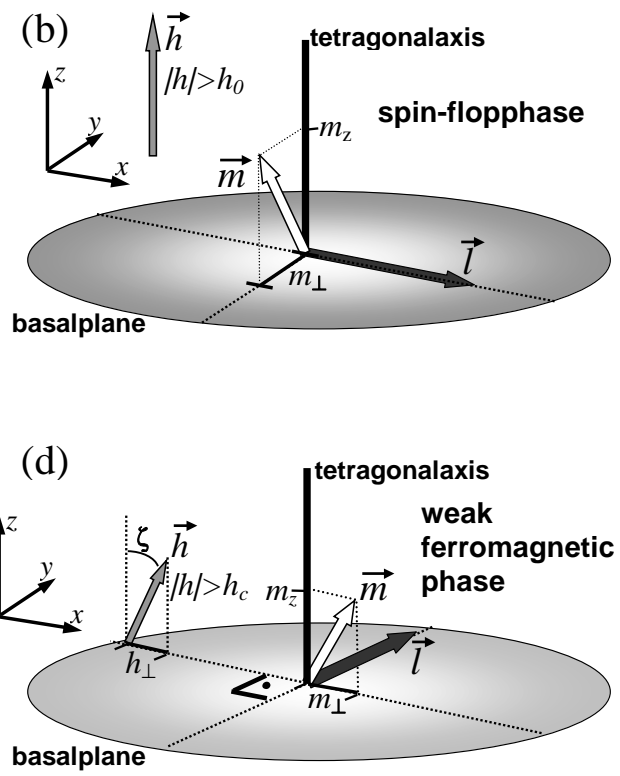

FIG. 1: Basic spin configurations described by the staggered magnetization $\mathbf{l}$ and the total magnetization $\mathbf{m}$ (for clarity, $|\mathbf{m}| \ll|\mathbf{l}| \simeq 1$ is not obeyed in the pictures) in homogeneous states of easy-axis systems $(K>0)$.

$(a)$ and $(b)$ external field $\mathbf{h}$ along the tetragonal axis. (a) Collinear or antiferromagnetic (AF) phase with $\mathbf{l} \| O Z$ and zero magnetization exists in the applied field along the tetragonal axis $0 \leq h \leq h_{0}$.

(b) Spin-flop phase with $\mathbf{m} \| O Z$ and with $\mathbf{l}$ in the basal plane is an important particular case of the weak ferromagnetic (WF) phase for $h>h_{0}$ along the tetragonal axis.

$(c)$ and $(d)$ magnetic field with oblique direction. The low symmetry canted phase $(c)$ exists for $\nu<1(16)$ and continuously transforms into the WF phase $(d)$ at the critical line $\nu=1$. In the canted phase the staggered magnetization is in the plane perpendicular to the the in-plane component of the applied field and all components of the vector $\mathbf{m}$ have generally nonzero values (cf. eg. (17). (d) General case of the WF phase. The staggered magnetization 1 lies in the basal plane, the component of the total magnetization along the tetragonal axis $\left(m_{z}\right)$ is induced by the corresponding component of the applied field, the in-plane components are due to the homogeneous Dzyaloshinskii-Moriya interaction and in-plane components of magnetic field. In easy-plane systems $(K<0)$ only the WF phase is stable for all values of the applied field.

\section{A. Homogeneous states}

The homogeneous states are described by the behaviour of the energy functional (16). Depending on the sign of $K$ the energy (16) describes two different types of antiferromagnetic ordering.

\section{1. $K>0 \quad$ Easy-axis system}

At zero field and in a magnetic field along the tetragonal axis for $h<h_{0}$ the antiferromagnetic phase with $\mathbf{l} \| z$-direction and $\mathbf{m}=0$ has the lowest energy. This magnetic structure is sketched in Fig. 1(a). At the field $h_{0}=\sqrt{K}$ the vector 1 "flops" down onto the basal plane. This is a so-called spin-flop transition. In the resulting spin-flop phase with $\theta=\pi / 2$, the total magnetization under influence of the homogeneous Dzyaloshinskii-Moriya interaction is slightly inclined from the tetragonal axis (Fig. 1 $1(\mathrm{~b}))$. In the region where $|\mathbf{m}| \ll 1$ the components of $\mathbf{m}, m_{z}=h / \lambda,\left|m_{\perp}\right|=d / \lambda$, are obtained from
(7). In the region $h_{0}<h<\lambda$ the total magnetization increases linearly with increasing field and finally at the "exchange" field $h_{\mathrm{ex}}=\lambda$ the spin-flop phase continuously transforms into the saturated "paramagnetic" phase with $|\mathbf{m}|=1, \mathbf{l}=0$ by a spin-flip transition. Note, in the spin-flop phase the magnetic state has an infinite degeneracy with respect to rotation of the vector $\mathbf{l}$ around the tetragonal axis. In-plane anisotropy reduces the degeneracy to certain preferable directions related by symmetry in the basal plane. E.g., in the case of fourth-order tetragonal anisotropy there are two mutually perpendicular directions of "easy" magnetization. In an increasing magnetic field deviating from the tetragonal axis the staggered magnetization 1 rotates to the basal plane in the plane perpendicular to the projection of $\mathbf{h}$ onto the basal plane. The angle $\theta$ between the vector 1 and the $z$-axis is $\sin \theta=\nu$ for $\nu<1$ (16). We name this state with a finite angle between staggered magnetization and $z$-axis canted phase (Fig. 11(c)). Finally, at the critical line $h_{c}\left(h_{\perp}, h_{y}\right)$ (Fig. 2) where $\nu$ from expression (16) attains the critical value $\nu=1$, a phase transition occurs into a phase with the staggered vector 


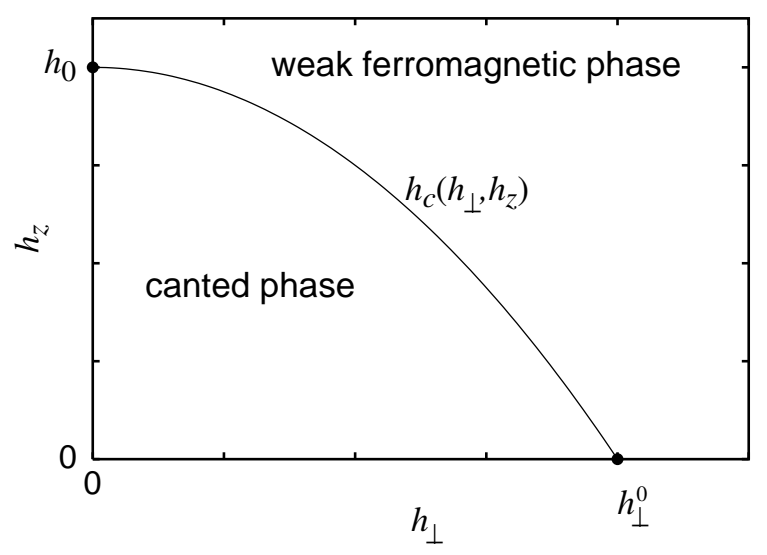

FIG. 2: $\left(h_{\perp}, h_{z}\right)$ phase diagram for the homogeneous states in easy-axis systems $(K>0)$. The low symmetry canted phase transforms into the WF phase by a second order phase transition at the critical line $h_{c}\left(h_{\perp}, h_{z}\right)$ determined by the condition $\nu=1$ in (16). Scales are given by $h_{0}=K^{1 / 2}$ and $h_{\perp}^{0}=K / d$.

lying in the basal plane, $\sin \theta=1$, and perpendicular to the applied field (Fig. 1 $1(\mathrm{~d})$ ). This is a weak ferromagnetic (WF) phase. The phase-diagram for this transition between canted and WF phase is depicted in Fig. 2. The total magnetization is deduced by substituting the equilibrium values of $\mathbf{n}=\mathbf{l} /|\mathbf{l}|$ into eq. (7). Assuming that the applied field is in the $X O Z$-plane, then the staggered magnetization rotates in the $Y O Z$-plane. From (7) the following expressions for the magnetization components result

$$
\begin{aligned}
& m_{x}=(h \sin \zeta+d \sin \theta) / \lambda, \\
& m_{y}=-h \cos \zeta \sin \theta \cos \theta / \lambda, \\
& m_{z}=h \cos \zeta \sin ^{2} \theta / \lambda,
\end{aligned}
$$

where $\theta=\arcsin \nu$ for the canted phase and $\theta=\pi / 2$ in the weak-ferromagnetic phase.

The relative orientation of the vectors $\mathbf{l}$ and $\mathbf{m}$ is fixed by the sign of the constant $d$. This handedness of the magnetic structures reflects the chiral character of the interaction (1) and leads to the nonequivalence of energies for the states with antiparallel values of $\mathbf{l}$ in oblique magnetic fields. One can understand this considering the shapes of the potential $\Phi(16)$ with applied fields (Fig. 3). In the general case (Fig. 3(a)) of an oblique field, i.e. an applied field deviating from the tetragonal axis, the antiferromagnetic phase with $\theta=\pi n$ transforms into the WF phase $\left(h>h_{c}\right)$ via the canted phase. From the potential profile for this canted phase (Fig. 3(a), $0<h<h_{c}$ ) one immediately sees for a given state that the corresponding state with antiparallel orientation of $\mathbf{l}$ has a different energy and generally is not an equilibrium state. Thus, the symmetry between states with antiparallel $\mathbf{l}$ peculiar to ordinary antiferromagnetic phases is violated. The stable states in this canted phase are separated by two types of potential barriers. At the transition into the
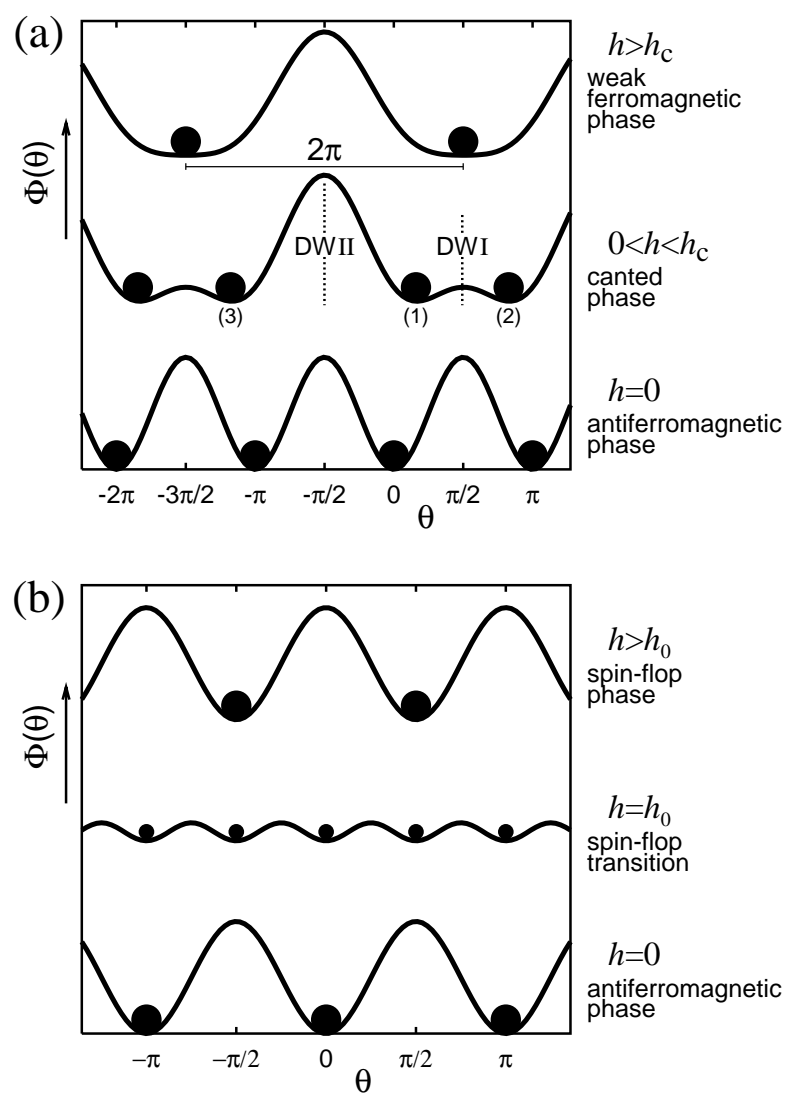

FIG. 3: Schematic evolution of the potential profiles $\Phi(\theta)$ under influence of the applied field for easy-axis systems $(K>0)$ in an oblique field (a) and in a field in direction of the tetragonal axis (b).

In (a) for the canted phase two different barriers occur between equivalent equilibrium states. Different domain walls correspond to these barriers: DW I between state (1) and (2), and DW II between (1) and (3).

WF phase the lower potential barrier disappears and the higher separates states with $\theta=\pi / 2+2 n \pi$. In a magnetic field along the tetragonal axis (Fig. B(b)) the potential barriers in $\Phi(16)$ disappear as the field approaches the spin-flop field from both sides, i.e. in the antiferromagnetic $\left(h<h_{0}\right)$ and in the spin-flop phases $\left(h>h_{0}\right)$. This means that at the transition field $h=h_{0}$ the potential barriers between coexisting antiferromagnetic and spinflop states are anomalously low and are determined by the values of the fourth-order anisotropy.

\section{2. $K<0 \quad$ Easy-plane system}

In the ground state for easy-plane systems $K<0$ the staggered magnetization lies in the basal plane with a spontaneous magnetization, $|\mathbf{m}|=d / \lambda$, perpendicular to the vector $\mathbf{n}$. Therefore, this is a weak-ferromagnetic phase. The behaviour of the system under influence of 


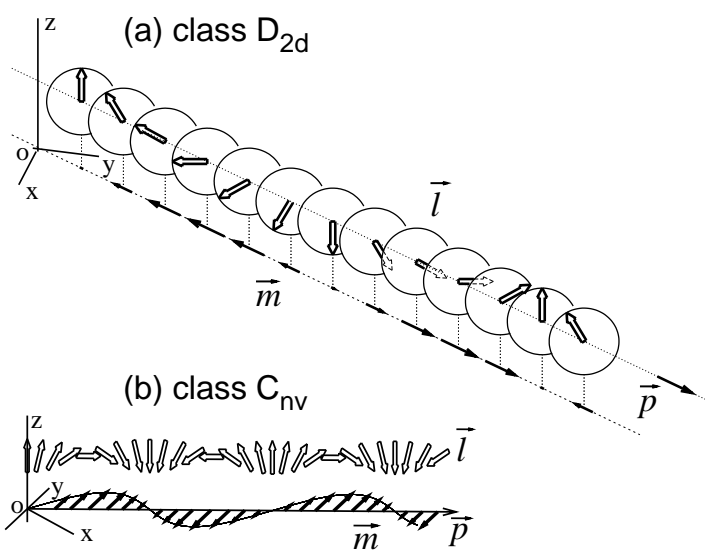

FIG. 4: Basic modulated structures (a) - helicoid for systems with $D_{2 d}$ symmetry; (b) - cycloids for antiferromagnets with $C_{n v}$ symmetry.

a magnetic field is similar to that in the easy-axis system (section III A 1) for $h_{z}$ larger than the spin-flop field, namely the vector $\mathbf{l}$ is oriented perpendicular to $\mathbf{h}$, and the total magnetization gradually increases in increasing field.

\section{B. Helical structures}

The equations minimizing the functional (10) also permit solutions with chiral modulations propagating in the basal plane. First we consider structures modulated along a certain fixed direction in the basal plane and homogeneous perpendicular to this direction. This yields one-dimensional spirally modulated states comprising helicoids and cycloids. In the absence of inplane anisotropy all propagation directions are equivalent. The structure of these modulated states depends on the crystal symmetry which manifests itself in different functional forms of the Lifshitz invariants (see (5), (6)). For antiferromagnets belonging to the crystallographic class $D_{2 d}$ the staggered vector $\mathbf{l}$ rotates in the plane perpendicular to the propagation direction, i.e. $\psi=\pi / 2$. These states are helicoids (Fig. [1(a)). This rotation of 1 reminds the behaviour of the magnetization vector for Bloch walls in ferromagnets (see 33 ). In the case of $C_{n v}$ symmetry, $\mathbf{l}$ rotates in the plane formed by the tetragonal axis and the propagation direction $(\psi=0)$ forming cycloids (Fig. G(b)). This is akin to Néel domain walls in ferromagnets. The rotation in the spirals has a fixed sense determined by the condition that the inhomogeneous Dzyaloshinskii-Moriya energy must be negative. In both cases, the spirals are accompanied by oscillations of the magnetization $\mathbf{m}$ perpendicular to the plane of rotation according to (7) (Fig. (1). In zero field, $m=d \sin \theta / \lambda$. Under the influence of an applied magnetic field the spirals orientate in such a way that the rotation of $\mathbf{l}$ occurs in the plane perpendicular to the projection of $\mathbf{h}$

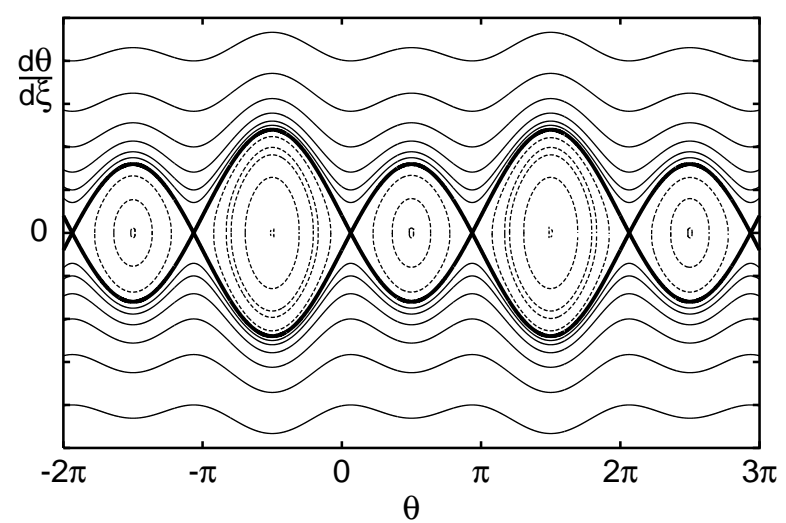

FIG. 5: Typical phase portrait of the solutions for the equation (19) in the canted phase. The separatrix curves between open (continuous lines) and closed (dashed lines) orbits are highlighted by a thick line.

onto the basal plane. As above for the homogeneous structures, we assume that the magnetic field lies in the $X O Z$-plane and the vector $\mathbf{n}$ in the $Y O Z$-plane. For helicoids $\left(D_{2 d}\right.$ symmetry) the propagation direction is along the $x$-axis and for cycloids $\left(C_{n v}\right.$ symmetry) along the $y$-axis. The spatial coordinate along the propagation direction is measured in reduced units of $x_{0}$ according to (14), $\xi=x_{i} / x_{0}$. In these reduced units the energy functional (10) for one-dimensional modulations assumes the form

$$
\widetilde{\widetilde{W}}=\sqrt{\frac{A|K|}{\lambda}} \int\left\{\left(\frac{d \theta}{d \xi}\right)^{2}+\Phi(\theta)+\frac{4 D}{\pi D_{0}}\left(\frac{d \theta}{d \xi}\right)\right\} d \xi,
$$

where the multiplicative constants due to the integrations in directions of $z$ and perpendicular to $\xi$ are absorbed in $\widetilde{\widetilde{W}}$. $\Phi(\theta)$ is given by (16) and $D_{0}$ by (14). The first integral of the Euler equation for the functional (18) is readily derived,

$$
\left(\frac{d \theta}{d \xi}\right)^{2}-\Phi(\theta)=E
$$

where $E$ is an integration constant. In passing, we remark that the Euler equation with the potential $\Phi(\beta)$ from (16) is related to the double sine-Gordon equation. 35

Typical phase trajectories of $(19)$ in the $\left(\theta_{\xi}, \theta\right)$-phase plane are plotted in Fig. 5 (here, we use the abbreviation $\left.\theta_{\xi} \equiv d \theta / d \xi\right)$. The separatrices obtained for $E=0$ cross each other in the points corresponding to the minima of the function $\Phi(\theta)$. They divide the phase plane into regions with closed $(E<0)$ and open $(E>0)$ trajectories (Fig. 5). The closed trajectories correspond to alternating rotation of the staggered vector and obviously are not of interest for our model as they describe inhomogeneous states with alternating sense of rotation that do not minimize the energy related to the inhomo- 


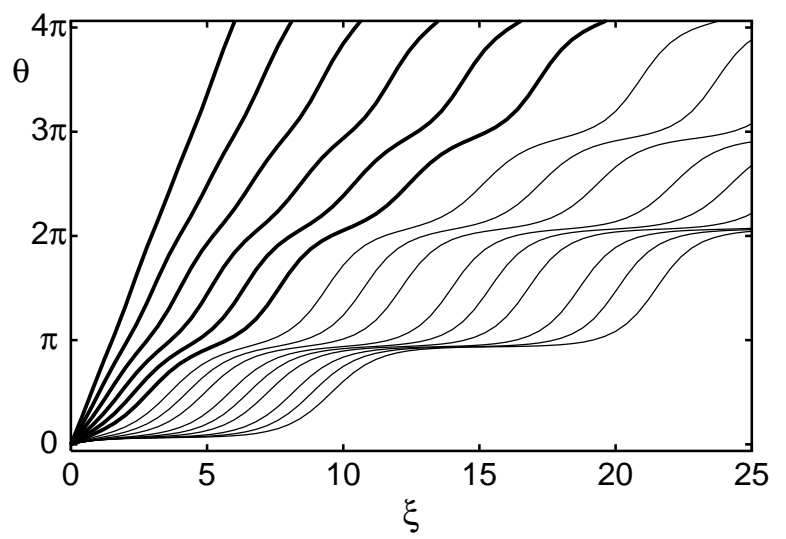

FIG. 6: Series of solutions of $(19) \theta(\xi, E)$ corresponding to the open orbits in Fig. 5. Solutions shown by thinner lines correspond to open orbits with $E>0$ close to the separatrix curve of Fig. 5 (not shown there).

geneous Dzyaloshinskii-Moriya interactions. The modulated states with fixed rotation sense are described by open trajectories. The integration of eq. (19) with $\Phi(\theta)$ from (16) yields the set of solutions $\theta(\xi, E)$ parametrized by the constant $E$ (Fig. 6). These solutions can be expressed analytically as certain cumbersome combinations of elliptic functions 36 (see, e.g., Refs. 0.30.34). Here, for simplicity, we derive representative solutions by direct numerical integration of (19). Using (19) the energy density $\bar{w}$ averaged over a period $\Xi$ of a modulated state can be written as functions of the parameter $E$

$$
\begin{gathered}
\bar{w}=\frac{1}{\Xi(E)} \int_{0}^{2 \pi} \frac{[E+2 \Phi(\theta)] d \theta}{\sqrt{\Phi(\theta)+E}}-\frac{2 \pi D}{\Xi(E)}, \\
\Xi(E)=\int_{0}^{2 \pi} \frac{d \theta}{\sqrt{\Phi(\theta)+E}} .
\end{gathered}
$$

Note, eq. (19) does not involve contributions from the inhomogeneous chiral Dzyaloshinskii-Moriya interactions and, thus, its solutions (Fig. 6) do not depend on these chiral interactions. Therefore, these solutions (Fig. 6) have the same functional form as those for the corresponding model with $D=0$ (centrosymmetric systems). However, the energy $\bar{w}(20)$ of the system depends on the contribution from the Lifshitz invariants. This energy has different values for different integral curves $\theta(\xi, E)$. The equation $d \bar{w} /(d E)=0$ to derive the optimal values $\tilde{E}$ can be reduced to the following form

$$
\int_{0}^{2 \pi} d \theta \sqrt{\Phi(\theta)+E}=4 D / D_{0} .
$$

Hence, the spiral structure described by the integral curve $\theta(\xi, \tilde{E})$ obeying (21) corresponds to the equilibrium magnetic structure realized in a noncentrosymmetric system, where inhomogeneous Dzyaloshinskii interactions $w_{D}$ are operational. From the solutions of eqs. (19) and (21) the other equilibrium parameters of the spiral structures are readily calculated. In particular, eq. (20) yields the period of the structure $\Xi$, and the oscillating components of the vector $\mathbf{m}$ are expressed via (7) as functions of $\theta(\xi, \tilde{E})$. Depending on the ratio $D / D_{0}$ for the relative strength of the chiral interactions the modulated structures display the following characteristic evolution: For strong chiral interactions $D / D_{0} \gg 1$ the influence of the energy (16) is negligible and in the equilibrium states the staggered vector rotates with an essentially fixed "velocity" $\theta_{\xi}$ corresponding to the phase trajectories with $\theta_{\xi}=2 D /\left(\pi D_{0}\right)$. Perturbations of the uniform rotation for the spirals are related to the shape of the potential profiles (cf. Fig. [4 and the open trajectories in Fig. [2). Thus, the functional dependencies of $\theta(\xi, \tilde{E})$ contain important information on internal interactions of a system. When values of the parameter are smaller, $D / D_{0} \simeq 1$ the influence of the potential $\Phi(\theta)$, which determines preferable orientations in the crystal, violates uniform rotation of the staggered magnetization $\mathbf{l}$ in a spiral. Further weakening of the chiral interactions leads to "pinning" of $\mathbf{l}$ along certain ("easy") directions and squeezes the regions with "disadvantageous" orientations of the vector 1 . This tendency results in the formation of structures consisting of large domains with homogeneous states separated by narrow transition regions in which the vector $\mathbf{l}$ rotates from one easy direction to another similar to domain walls. Finally, for a certain critical value of $D$ the modulated phase is transformed into the homogeneous state. This transition is signalled by an unlimited growth of the period for the modulated state. In the phase space, states at this transition into the homogeneous state correspond to the separatrix (Fig. 5) which describes a set of isolated domain walls, i.e. walls with infinite separation between them. The finite stiffness of the exchange interaction prevents a complete annihilation of the domain walls and they may exist with finite thickness within homogeneous states as metastable topologically stable objects acting as nucleation centers during a reversal transition from the homogeneous into the modulated state.

Finally, it should be stressed that eqs. (19) and (21) provide general and rigorous solutions for onedimensional modulated structures in magnets with Lifshitz invariants of type (2) with arbitrary functional form for the potential $\Phi(\theta)$ in the functional (18). The above described evolution of the modulated states is not restricted to any particular form of $\Phi(\theta)$; the qualitative picture of this evolution rather has universal character for physically reasonable choices for $\Phi(\theta)$. Particular cases for such chirabspirals have been investigated starting from the papent for serpral groups of helical ferroand antiferromagnets. $12,03,30.37$

\section{Stability limits of the modulated states}

At the transition into homogeneous states the chiral spirals disintegrate into a system of noninteracting pla- 
nar domain walls. Such a transition can be found by comparing the energy of the chiral spiral to the energy of domain walls separating regings with different homogeneous states of the system.155 30 Below in section IV, we will discuss domain walls as topological defects in the magnetic system which require an excitation energy. Here, we are concerned with the competition between homogeneous and modulated equilibrium states. Then, a gain of energy through proliferation of domain walls indicates the instability of homogeneous states compared to a modulated state.

Let us consider a planar isolated domain wall between two infinitely extended regions with different spatially homogeneous magnetic structures that are described by the functional (16), i.e. equilibrium states in Fig. 3. The equilibrium structure of this isolated wall is determined by solving (19) with the boundary conditions $\left.\theta\right|_{\xi= \pm \infty}=\theta_{1,2},(d \theta / d \xi)_{\xi= \pm \infty}=0$, where $\theta_{1,2}$ are homogeneous configurations determined by $\Phi_{\infty} \equiv \min [\Phi(\theta)]=\Phi\left(\theta_{1}\right)=\Phi\left(\theta_{2}\right)$ for the functional (16). The direct integration of (19) yields the following results for the dependence of $\theta(\xi)$ in the wall and for the domain wall energy $\sigma$ (see 1938 )

$$
\begin{gathered}
\xi-\xi\left(\theta_{1}\right)=\int_{\theta_{1}}^{\theta} \frac{d \theta^{\prime}}{\sqrt{\left[\Phi\left(\theta^{\prime}\right)-\Phi_{\infty}\right]}}, \\
\sigma=\frac{\pi}{2} D_{0} \int_{\theta_{1}}^{\theta_{2}} \sqrt{\left[\Phi(\theta)-\Phi_{\infty}\right]} d \theta \pm D\left|\theta_{1}-\theta_{2}\right| .
\end{gathered}
$$

The function $\left[\Phi(\theta)-\Phi_{\infty}\right]$ is the deviation of the energy density (13) from the minimal value $\tilde{w}_{\infty}$ corresponding to the homogeneous states in adjacent domains. The first term in (23) is positive and represents increased energy contributions compared to those of the homogeneous states $\theta_{1}, \theta_{2}$. This increase is due to inhomogeneous exchange interactions and interactions included into the functional $\Phi(16)$. These defect energies are typical for the energy of magnetic domain walls.33 The second term is specific for noncentrosymmetric systems. Its sign is determined by the rotation sense of the staggered vector $\mathbf{n}$ in the domain wall. Clearly, for any sign of the constant $D$ there exists a rotation sense of the staggered magnetization leading to negative values for this energy contribution and, consequently, to a decrease of the domain wall energy. For sufficiently strong inhomogeneous chiral Dzyaloshinskii-Moriya interactions the total energy of the domain wall may be negative compared to the energy of homogeneous states. This manifests an instability of the homogeneous state with respect to chiral modulations. As already discussed above, such a transition takes place for coefficients $D$ larger than a certain threshold value necessary to overcome the positive energy contribution for inhomogeneous states due to the conventional magnetic interactions.

The wall energy (23) can be expressed via the height of the potential barrier, $\Delta \Phi=\max [\Phi(\theta)]-\Phi_{\infty}$, that sepa-
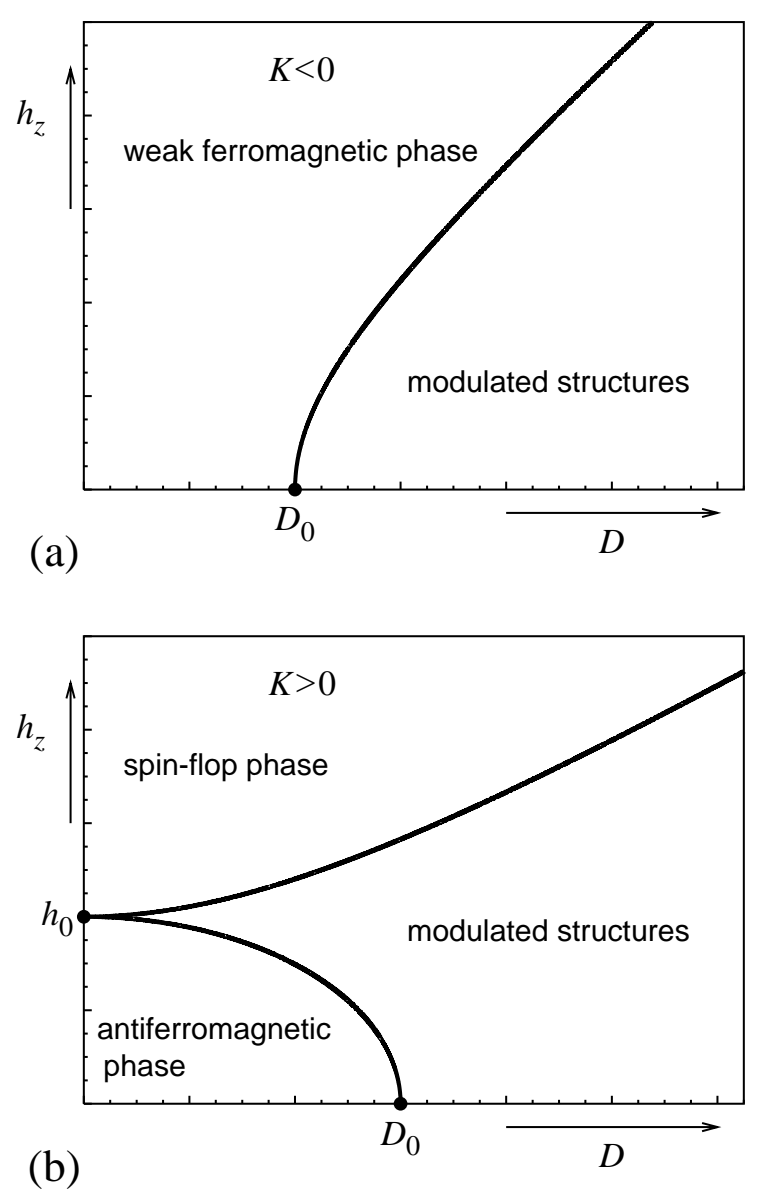

FIG. 7: Magnetic phases in the magnetic field along the tetragonal axis in dependence on strength $D$ of the inhomogeneous Dzyaloshinskii-Moriya interactions (a) $K<0$; (b) $K>0$. Note that for easy-axis antiferromagnets (b) in the vicinity of spin-flop field the modulated phases exists at arbitrarily small values of $D$.

rates the equilibrium states $\theta_{1}$ and $\theta_{2}$. This can be written in the following form

$$
\sigma=\left[\gamma D_{0} \sqrt{\Delta \Phi} \pm D\right]\left|\theta_{1}-\theta_{2}\right|
$$

where $\gamma$ is a numerical factor determined by the average value of the integrand in (23). These results have clear physical meaning. The higher the energy barrier $\Delta \Phi$ the stronger the chiral interaction necessary to overcome it and to stabilize modulated states. As was shown above, the potential profile $\Phi(\theta)$ (16) strongly depends on the strength and direction of the applied field (Fig. 3). Correspondingly, the critical values $D_{c}$ of the DzyaloshinskiiMoriya constant $D$ for transitions between homogeneous $\left(D<D_{c}\right)$ and modulated chiral states $\left(D>D_{c}\right)$ vary strongly with an applied magnetic field. The critical surfaces for these transitions in parameter space are given by the equation $\sigma=0$ using the functional (16). For 
easy-plane systems $(K<0)$ the critical surface is

$$
\frac{D_{c}}{D_{0}}=\sqrt{\left|\frac{h_{z}^{2}}{K}-1\right|}\left[\sqrt{1-\nu}-\frac{\nu}{2} \ln \left(\frac{\sqrt{1-\nu}+1}{\sqrt{1-\nu}-1}\right)\right] .
$$

This equation also describes the boundaries of the modulated states for easy-axis systems $(K>0)$ in magnetic fields larger than the spin-flop field $\left(h_{z}>h_{0}\right)$. For lower fields $\left(h_{z}<h_{0}\right)$ the equation

$$
\frac{D_{c}}{D_{0}}=\sqrt{1-\frac{h_{z}^{2}}{h_{0}^{2}}}\left[\sqrt{1-\nu^{2}}+\nu \arcsin \nu\right],
$$

gives the transition into the canted phase $(\nu \leq 1)$. Finally, the equation

$$
\frac{D_{c}}{D_{0}}=\sqrt{1-\frac{h_{z}^{2}}{h_{0}^{2}}}\left[\sqrt{\nu-1}+\nu \arcsin \sqrt{\frac{1}{\nu}}\right],
$$

describes the transition into the weak-ferromagnetic phase $(\nu>1)$. In particular, at zero field the critical value $D_{c}$ equals $D_{0}$. Thus, this constant is the lowest value for the Dzyaloshinskii constant $D$ to induce modulated ground states. The $\left(D, h_{z}\right)$ phase diagrams for an applied field in direction of the tetragonal axis are shown in Fig. 7 for the two cases $K>0$.

For easy-plane systems $(K<0)$ the critical surface $D_{c}\left(h_{\perp}, h_{z}\right)$ has a minimum in the origin with $D_{c}(0,0)=$ $D_{0}$ and monotonically increases with increasing magnetic field for any direction (Fig. \&(a)). When $D<D_{0}$ the chiral interactions are too weak to overcome the pinning due to the uniaxial easy-plane anisotropy. Then, the system exists in the homogeneous state with the staggered magnetization in the basal plane and a weak spontaneous magnetization (WF phase). For $D>D_{0}$ the WF phase becomes unstable. Under the influence of the inhomogeneous chiral interactions the vector $\mathbf{n}$ "escapes" from the basal plane and a chiral helix is formed. We add a remark about the peculiarity of this type of helix. In known easyplane systems with helical structures the magnetization (or the staggered magnetization in the case of antiferromagnets) rotates in the "easy-plane" and the propagation vector is perpendicular to this plane. In these noncentrosymmetric magnets such spirals are stabilized by Lifshitz invariants with gradients along the "hard-axis". In our model, however, the Lifshitz invariants include only gradients in the basal plane. Correspondingly, the chiral modulations in these systems have propagation directions only in the basal plane 11.15

For easy-axis systems $(K>0)$ the critical surface $D_{c}\left(h_{\perp}, h_{z}\right)$ has a more involved shape. In this case the lowest value of $D_{c}$ equals zero. This is reached at the spin-flop field $\left(0, \pm h_{0}\right)$ (Figs. B(b), \&(b)). Thus, near the spin-flop transition the modulated states arise at arbitrarily small values of $D$. This unusual situation is due to the particular evolution of the potential profile (16)
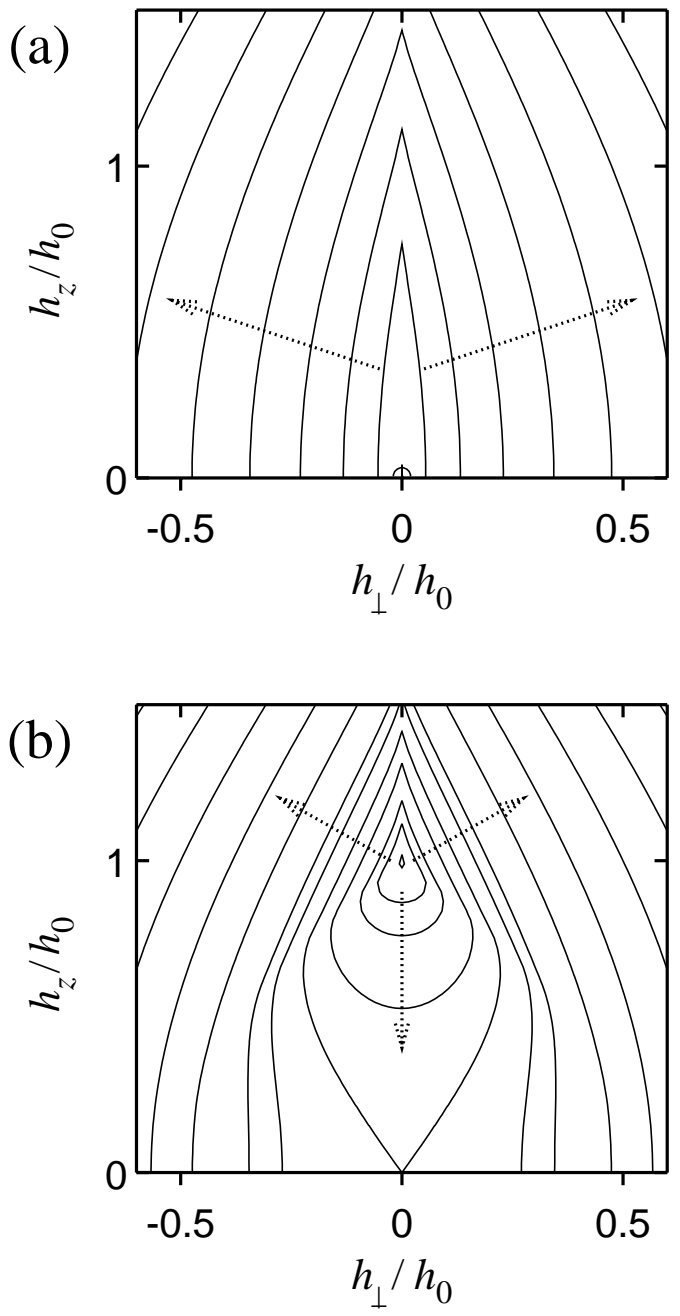

FIG. 8: Contour lines $D_{c}\left(h_{\perp}, h_{z}\right)=$ const of the critical surfaces for modulated states. (a) $K<0$ surface given by (25); (b) $K>0$ surface according to (25), (26), and (27). (The direction of increasing $D_{c}$ is indicated by dotted arrows.)

in a magnetic field directed along the tetragonal axis (Fig. 3(b)). In this case the uniaxial anisotropy and the applied magnetic field have competing influence on the magnetic structure. While the easy-axis anisotropy orientates the staggered magnetization along the tetragonal axis, the applied field orientates it perpendicular to this axis. An increasing magnetic field in the region $h<h_{0}$ gradually decreases the potential barrier between the states of the antiferromagnetic phase with antiparallel staggered magnetization (Fig. 3(b)). When the spin-flop is reached the applied field completely cancels the influence of uniaxial anisotropy, and the potential (16) equals zero for any orientation of the staggered magnetization. This infinite degeneracy of magnetic states is artificial because of the neglect of higher order anisotropy contributions. A fourth-order uniaxial anisotropy $K_{2} \sin ^{4} \theta$ removes this degeneracy. We may generally state that in 
noncentrosymmetric easy-axis antiferromagnets near the spin-flop field the potential barrier between minima (and, therefore, the critical values of $D$ ) is determined by the much weaker fourth-order anisotropy constant $K_{2}$. We add two remarks here: (i) For centrosymmetric easy-axis antiferromagnets (models I.) and II.) of subsection II A), the situation near spin-flop transitions is physically comparable and has been studied in detail.19.28 There, the relation between interactions and homogeneous magnetic states is well understood. (ii) In cubic helimagnets, 12 where magnetocrystalline anisotropy is represented only by fourth-order terms, no suppression of the modulated states has been observed, rather chiral modulations exist in the complete region of existence of magnetically ordered states. Estimates based on the physical origin of these magnetic energy contributions yield so weak threshold values that modulated chiral states-near the spin-flop field should be expected generally.30 Therefore, this competition between antisymmetric exchange and anisotropies makes the easy-axis noncentrosymmetric antiferromagnets particularly interesting systems for a search for and investigation of modulated chiral states.

\section{LOCALIZED CHIRAL STRUCTURES}

In this section we consider the influence of the Dzyaloshinskii-Moriya interactions on localized magnetic defects within homogeneous magnetic configurations. As there is a wide variety of possible defect structures in the different phases, we will present only a few examples to demonstrate the general principles which rule defect structures for the noncentrosymmetric antiferromagnets under the influence of chiral couplings. A formal mathematical description of isolated, planar one-dimensional defect structures, i.e. domain walls, was already developed above in section IIIC. In the next subsection, we discuss physical importance and properties of such domain walls for antiferromagnetic systems described by our model. Subsection IVB is devoted to linear twodimensional defect structures, i.e. vortices.

\section{A. Domain walls or kinks}

Planar defects (domain walls or kinks) are commonly observed magnetic localized states in many classes of antiferromagnetic materials. 39 They separate homogeneous states with different degenerate directions of the staggered magnetization. An example is provided by $180-$ degree domain walls between regions with antiparallel staggered magnetization, i.e. different antiferromagnetic phases, in easy-axis antiferromagnets. In the antiferromagnets under discussion, the rotation of the vector $\mathbf{n}$ within a domain wall is accompanied by oscillation of the total magnetization. The spin arrangement in such domain walls is similar to that in the corresponding spirals (Fig. 4). Rotation of $\mathbf{n}$ as in a Bloch wall with longi-

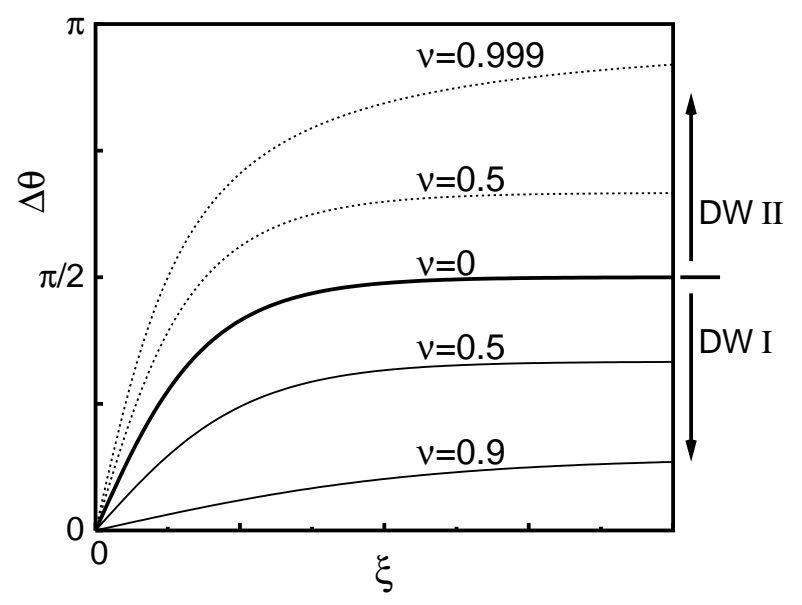

FIG. 9: Structure of domain walls in the canted phase given by the turn angle $\Delta \theta$ as function of distance from the wallcenter $\xi=0$ for different values of the parameter $\nu$. The 180 -degree domain walls of the $\operatorname{AF}$ phase $(\nu=0)$ are deformed in the canted phases either into walls with a decreased value of $\Delta \theta(\xi \rightarrow \infty)$ (DW I, profiles with continuous lines) or an increased $\Delta \theta(\xi \rightarrow \infty)$ (DWII, profiles with dashed lines). DW II are transformed into 360-degree walls in the limit $\nu=1$.

tudinal modulation of the vector $\mathbf{m}$ (Fig. 国(a)) should occur in noncentrosymmetric antiferromagnets belonging to crystallographic class $D_{2 d}$. Néel-wall-like structures with transversal oscillation of the magnetization correspond to the noncentrosymmetric antiferromagnets from class $C_{4 v}$ (Fig. 4(b)). The inhomogeneous chiral Dzyaloshinskii-Moriya interactions do not influence the structure of the domain walls (see above, remarks following eq.(201)). However, the domain wall energies do depend on the rotation sense according to (24). As discussed above, the modulated structures have a fixed sense of rotation that corresponds to a decrease of total energy compared to the homogeneous states. Spirals with opposite sense of rotation are unstable (even with respect to the homogeneous states) and never arise in real systems. Contrary to this, domain walls with disadvantageous sense of rotation, although increasing the energy, should be found within these antiferromagnets with similar probability because domain walls in antiferromagnets have mostly "kinetic" origin in contrast to domain structures in ferromagnets, i.e. antiferromagnetic domain structures are formed during the transition to the ordered states or as a result of reorientation transitions. These processes are largely independent of domain wall energies.

The structure of such domain walls can be derived by integration in (22). Here, we restrict ourselves to one example of a practical calculation. We obtain the structure and characteristic parameters of the domain walls in the canted phase (see Figs. 1, 2). Within all regions of their existence $(\nu \leq 1)$ the equilibrium states are sep- 


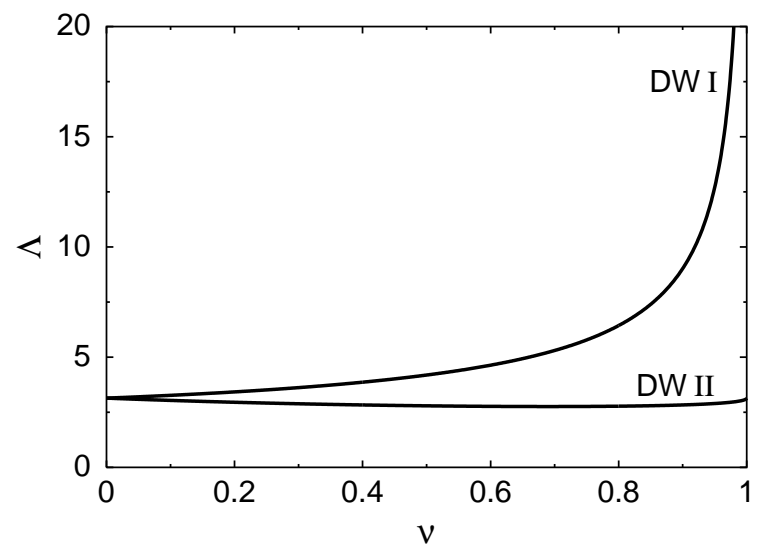

FIG. 10: The domain wall widths $\Lambda$ as functions of the parameter $\nu$ for the two wall types in the canted phase, (a) DW I (b) DW II, according to (29).

arated by two types of barriers in the potential profile $\Phi(\theta)$ (Fig. 3(a)). Correspondingly there exist two types of domain walls in the canted phase. The first low energy domain wall (DW I) separates homogeneous states with $\theta=\arcsin \nu$ and $\theta=\pi-\arcsin \nu$; and DW II corresponding to the higher potential barrier separates states with $\theta=\arcsin \nu$ and $\theta=-\pi-\arcsin \nu$ (Fig. 3(a)). Evaluating the integral (22) with $\Phi$ from (16) yields the following results

$$
\sin \theta=\frac{\nu \cosh \left(\xi \sqrt{1-\nu^{2}}\right) \pm 1}{\cosh \left(\xi \sqrt{1-\nu^{2}}\right) \pm \nu}
$$

Wall structures for both domain wall types in the canted phase with varying $\nu$ are displayed in Fig. 9. The effective thickness of domain walls $\Lambda$ is usually determined as a distance between points where the tangent at the inflection point intersects the lines $\theta=\theta_{1}$ and $\theta=\theta_{2}$. 33 For our example this definition yields the following expression

$$
\Lambda=\left|\theta_{1}-\theta_{2}\right|\left(\frac{d \theta}{d \xi}\right)_{\xi=0}^{-1}=\frac{\pi \mp 2 \arcsin \nu}{1 \mp \nu} .
$$

(In $(28)$ and $(29)$ the upper/lower signs correspond to DW I/DW II.) The dependence of the wall thickness $\Lambda$ on $\nu$ for both types of walls is shown in Fig. 10 . For increasing $\nu$ the difference between magnetic configurations in the adjacent domains separated by DW I $(\Delta \theta=\pi-2 \arcsin \nu)$ and the potential barrier $(\Delta P h i=$ $\left.\Phi(\pi / 2)-\Phi\left(\theta_{1}\right) \sim(1-\nu)^{2}\right)$ gradually decreases while the thickness of the wall increases. At the critical point of the transition into the weak ferromagnetic phase, $\nu=1$, the difference between magnetic states in the domains disappears and the wall spreads out without bounds. For DW II the potential barrier $\left.\Delta \Phi \sim(1+\nu)^{2}\right)$ and $\Delta \theta=\pi+2 \arcsin \nu$ increases with increasing $\nu$. At the critical point, $\nu=1$, these walls transform into 360 degree domain walls (Figs. 9 and 10).
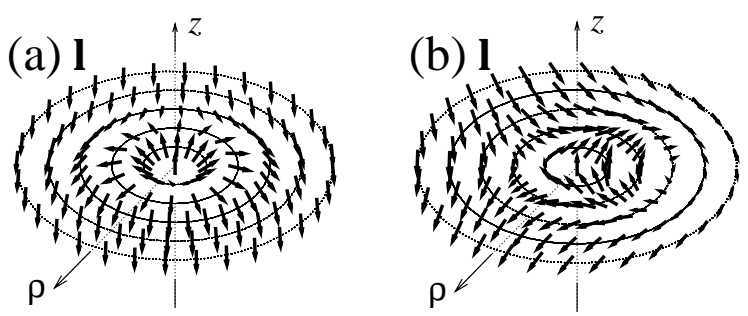

(c) 1

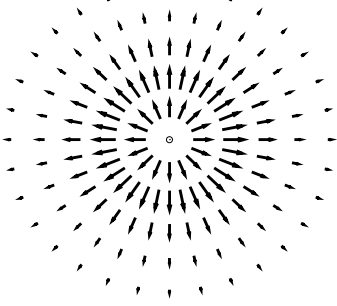

(d) 1

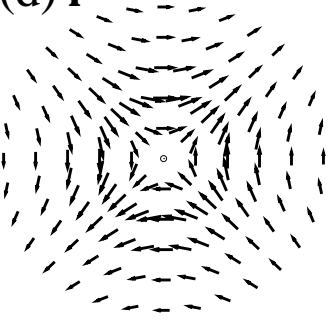

(e) $\mathbf{m}$

(f) $\mathbf{m}$
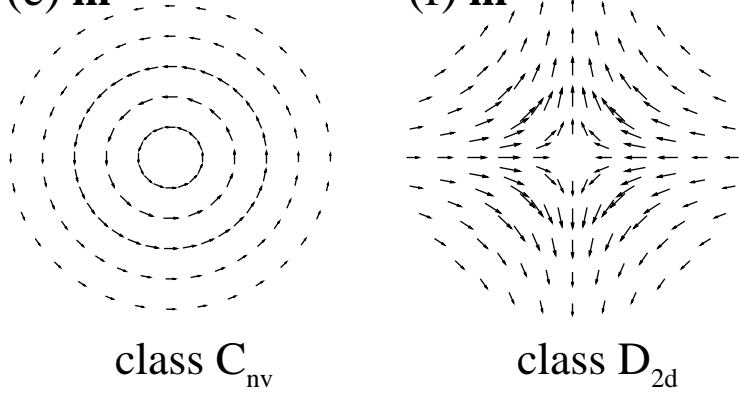

FIG. 11: Vortex structure for antiferromagnets with $C_{n v}$ symmetry (a), (c),(e) and $D_{2 d}$ symmetry (b), (d), (f). (a) and (b) distributions of staggered vector l. (c) - (f) projections of $\mathbf{l}$ and oscillating total magnetization $\mathbf{m}$ in the basal plane, respectively.

Structures and parameters for 180-degree domain walls in the antiferromagnetic and spin-flop phases can be derived in a similar way. All these domain walls may play the role of nucleation centers during the transition from the homogeneous to modulated states. On the other hand, as demonstrated in the previous section, at a transition into the homogeneous state the spiral states break down into a system of isolated plane walls.

\section{B. Vortices or skyrmions}

Linear magnetic defects are another type of topological excitations that can exist in noncentrosymmetric magnetic crystals due to the stabilizing effect of the inhomogeneous Dzyaloshinskii-Moriya interactions 15 At zero magnetic field and in fields applied along the tetragonal axis the model (3) is invariant to rotation about the $z$-axis. Solutions for the vector $\mathbf{n}(\mathbf{r})$ axially symmetric in the basal plane and uniform along the tetragonal 


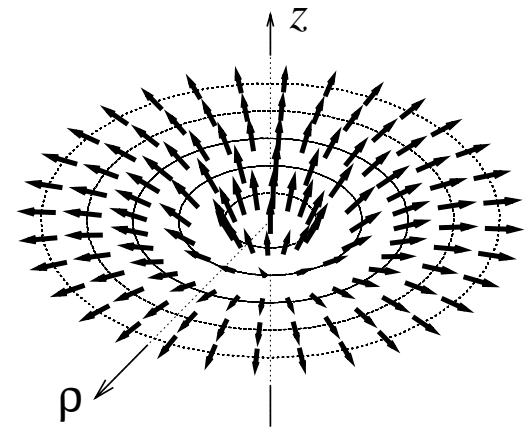

FIG. 12: Delocalized vortex structure in the spin-flop phase in crystals with $C_{n v}$ symmetry. Staggered vector $\mathbf{l}$ is shown.

axis, i.e. vortices, obey this symmetry. As an example of such localized states we consider an isolated vortex in the antiferromagnetic phase $(K>0, \mathbf{h}$ is parallel to the tetragonal axis and smaller than the spin-flop field $\left.h<h_{0}\right)$. We assume that the staggered magnetization is oriented parallel to the $z$-axis on the vortex axis and rotates into the antiparallel orientation with increasing radial distance from the vortex core. It is convenient to introduce cylindrical coordinates for the spatial variables, $\mathbf{r}=x_{0}(\rho \cos \varphi, \rho \sin \varphi, z)$, in the expression for the energy (10). (As earlier in the case of the spirals, we use length units $x_{0}=\sqrt{A \lambda /|K|}$.) The analysis of the energy functional (10) shows that the problem has solutions $\theta(\rho)$ with

$$
\psi=\varphi \text { for class } C_{n v}, \quad \psi=\pi / 2-\varphi \text { for class } D_{2 d} .
$$

For $C_{n v}$ symmetry the solution (30) describes a cycloidlike rotation of the staggered magnetization vector $\mathbf{l}$ (Figs. 11(a) and (c)). In the case of $D_{2 d}$ symmetry the vortex has more a sophisticated structure (Figs. 11(b) and (d)). The rotation of the staggered magnetization in the vortices is accompanied by in-plane oscillations of the total magnetization (Figs. 11(e),(f)) as described by eq.(7).

The equilibrium distribution $\theta(\rho)$ is determined from the differential equation common for both classes

$$
\begin{aligned}
\frac{d^{2} \theta}{d \rho^{2}}+ & \frac{1}{\rho} \frac{d \theta}{d \rho}-\frac{\sin \theta \cos \theta}{\rho^{2}} \\
& +\frac{4 D}{\pi D_{0}} \frac{\sin ^{2} \theta}{\rho}-\left(1-\frac{h^{2}}{h_{0}^{2}}\right) \sin \theta \cos \theta=0
\end{aligned}
$$

with boundary conditions $\theta(0)=0$ and $\theta(\infty)=\pi$ for localized vortices in the antiferromagnetic phase $\left(h<h_{0}\right.$, Fig. 11), or $\theta(\infty)=\pi / 2$ for delocalized vortices in the spin-flop phase $\left(h>h_{0}\right.$, Fig. 12). These different boundary conditions result in an important physical difference between these two cases of antiferromagnetic vortices: For the localized vortices the homogeneous equilibrium state is established everywhere for $\rho \rightarrow \infty$ and the inhomogeneity is localized in the vortex core. In the case

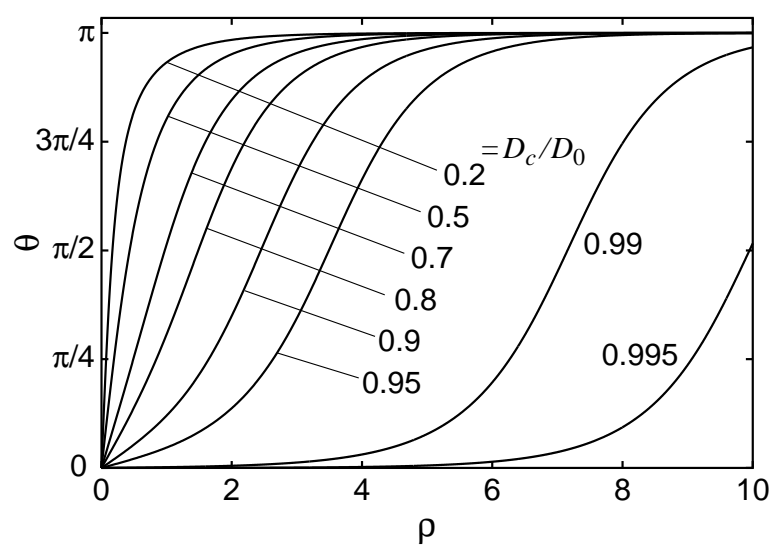

FIG. 13: Vortex profiles $\theta(\rho)$ : solutions of 31 for different values of $D / D_{0}$ in zero applied field.

of the spin-flop phase, the vortex structure at $\rho \rightarrow \infty$ is inhomogeneous with $\theta=\pi / 2$, but the angle $\psi$ rotates through the a full circle from 0 to $2 \pi$. Therefore, these vortices are named delocalized. Eq. (31) has solutions only when $D$ is smaller than the critical values for the transition to the modulated phase $D_{c}(h)$ given by (26). Typical solutions for $\theta(\rho)$ are plotted in Fig. 13. As $D$ approaches the critical value $D_{c}$ the vortex expands without bounds. Eq. (31) functionally coincides with the equations for isolated vortices in other models with Lifshitz invariants, i.e. models for noncentrosymmetric ferromagnets, 15.40 for other classes of antiferromagnets, 30 as well as for chiral liquid crystals.41 For detailed analysis of eq. (31) and discussion of the related questions see these papers.

The vortices or skyrmions considered here (Figs. 11, 12, 13) are non-singular linear defect structures. They belong to topological defects studied in many fields of the modern physics Similar topological objects arise in superfluid helium, 42 in two-dimensional electronic systems (Hall skyrmions), 43 or in nanomagnetic materials. 44 It is important to mention that there is a fundamental correspondence between these theoretical models. 45 In the isotropic case $\left(w_{D}=\tilde{w}=0\right.$ in (100) the equation for the vortex has analytical solutions which are well-knowm as Belavin-Polyakov-solutions for nonlinear $\sigma$-models. $\frac{16}{6}$ These solutions turned out to be unstable in centrosymmetric magnetic crystals and collapse spontaneously under the influence of anisotropic internal interactions or applied magnetic fields. Thus, the Lifshitz invariants are crucial for stabilizing these vortex structures in noncentrosymmetric magnetic crystals. ${ }^{2}$ Hence, such lowsymmetry magnetic crystals are interesting and important systems for investigations of general properties of vortices.

In the spin-flop phase the vortex states have delocalized character (Fig. 12). They are similar to vortex states in liquid helium or some textures in liquid crystals. 17 Such vortices for noncentrosymmetric antiferromagnets 
with $d=0$ have been investigated in Ref. 30 . They readily form localized vortex pairs similar to those responsible for Berezinskii-Kosterlitz-Thouless transitions. 18 An applied magnetic field deviating from the tetragonal axis violates the axial symmetry of the system. Then, the twodimensional localized states are expected to have various elongated shapes similar to those observed in chiral liquid crystals. 49 The Lifshitz invariants can also stabilize three-dimensional localized states (as free spherulites or drops).40 Up to now no experimental observations or theoretical investigations of such structures have been reported.

Concluding this section we draw attention to an important difference between the localized states in our model and those in other magnetic systems. In noncentrosymmetric antiferromagnets with weak-ferromagnetism due to the oscillating weak magnetization in the basal plane the domain walls and the vortices are susceptible to the influence of applied magnetic fields. For this reason, noncentrosymmetric antiferromagnets are convenient for the study of phase transformations and the dynamics of such nonlinear localized excitations.

\section{RELATION TO EXPERIMENTAL OBSERVATIONS}

The known noncentrosymmetric antiferromagnets with weak ferromagnetism include two tetragonal crystals. First, for $\mathrm{Ba}_{2} \mathrm{CuGe}_{2} \mathrm{O}_{7}$ belonging to the crystallographic class $\overline{4} 2 m\left(D_{-}\right)$, chiral modulations were discovered five year ago.16 For the crystallographic class $4 \mathrm{~mm}$ $\left(C_{4 v}\right)$ only the antiferromagnet $\mathrm{K}_{2} \mathrm{~V}_{3} \mathrm{O}_{8}$ has apparently been investiogated and no modulated states have been found yet 1850 Here, we shortly review experimental data for these two antiferromagnetic compounds within the framework of our theory. In the last subsection we comment on related experiments on noncentrosymmetric antiferromagnetic crystals.

\section{A. $\mathrm{Ba}_{2} \mathrm{CuGe}_{2} \mathrm{O}_{7}$}

A chiral spiral with propagation vector in the basal plane and period length of about 37 unit cells was found as magnetic ground state for $\mathrm{Ba}_{2} \mathrm{CuGe}_{2} \mathrm{O}_{7}$ (space group $\left.P \overline{4} 2{ }_{1} m\right)$. 16 It was also found that a rather strong magnetic field applied along the tetragenal axis induces a transition into a homogeneous state.17 The field dependencies of the period and the magnetization reported are in quantitative agreement with theoretical results of Ref. 17. It appears that there is no local minimum of the period length in dependence on the strength of a magnetic field applied along the tetragonal axis. This implies that the uniaxial anisotropy of this crystal is of easy-plane type $(K<0)$ (cf. Fig. 7). To analyze their experimental data the authors explored the model with $d=0$. In their experiments there is no indication of effects related to weak ferromagnetism.

Further detailed investigations in magnetic fields applied along other directions are required to determine the character of the uniaxial anisotropy and the values of the other characteristic parameters of the magnetic system within the general phenomenological expression (3) for the energy.

\section{B. $\mathbf{K}_{2} \mathbf{V}_{3} \mathbf{O}_{8}$}

For this compound, at a temperature of $2 \mathrm{~K}$ (the Néel temperature is about $4 \mathrm{~K}$ ) the magnetization curves in a magnetic field along tetragonal axis and in the basal plane indicate reorientation transitions. 18 These transitions are similar to those earlier observed in centrosymmetric antiferremagnets with weak ferromagnetism, e.g. hematite.24.27 The authors conclude from neutron diffraction experiment that there are no indications of chiral modulations. 18.50 According to the results of our theory such a situation may take place for easy-axis systems $(K>0)$ with weak chiral interactions $\left(D<D_{0}\right)$. As was discussed above, in the vicinity of the spinflop field the criterion for the stabilization of the modulated states is considerably weakened. Thus, the search for modulated states in this system should be started from thorough investigation near the spin-flop field. We add that there are two other similar noncentrosymmetric vanadium oxides $\mathrm{Rb}_{2} \mathrm{~V}_{3} \mathrm{O}_{8}$ and $\left(\mathrm{NH}_{4}\right)_{2} \mathrm{~V}_{3} \mathrm{O}_{8}$ which are supposed to possess antiferromagnetic order below $10 \mathrm{~K} 51$ They could be investigated in search for effects of chiral interactions.

\section{Other noncentrosymmetric antiferromagnets}

The copper metaborate $\mathrm{CuB}_{2} \mathrm{O}_{4}$ (space group $I \overline{4} 2 d$ $\left(D_{2 d}^{12}\right)$ ) belongs to the same noncentrosymetric class as $\mathrm{Ba}_{2} \mathrm{CuGe}_{2} \mathrm{O}_{7}$. However, according to 525354 it has a more sophisticated four-sublattice antiferromagnetic structure with in-plane anisotropy. A long-periodic modulated state has been observed in this crystal for a certain temperature range. 5354 Finally we mention here two other noncentrosymmetric antiferromagnets: a modulated chiral state has been observed in the noncentrosymmetric antiferromagnet $\mathrm{BiFeO}_{3}$ (space group R3c) 55 For $\mathrm{CuFeS}_{2}$ (space group $I \overline{4} 2 d\left(D_{2 d}^{12}\right)$ ) antiferromagnetic order was reported to exist, however, no details about the magnetic structures are given 56

\section{CONCLUSIONS}

In this paper we show that the novelantiferromagnetic crystals $\mathrm{Ba}_{2} \mathrm{CuGe}_{2} \mathrm{O}_{7} 16$ and $\mathrm{K}_{2} \mathrm{~V}_{3} \mathrm{O}_{8} 18$ in spite of the reported difference in their magnetic properties belong to a common, previously unknown class of magnetic crystals: noncentrosymmetric antiferromagnets with weak 
ferromagnetism. The phenomenological expression for the magnetic energy of such systems including all interactions allowed by symmetry (3) can be reduced to the functional (8) which describes the orientation of the staggered magnetization and can be considered as general model for two-sublattice antiferromagnets. It includes, as specific cases, all main classes of antiferromagnetic crystals (collinear antiferromagnets, antiferromagnets with weak ferromagnetism, noncentrosymmetric antiferromagnets without and with weak ferromagnetism). Further, by using realistic assumptions about the relative strengths of the phenomenological constants in (10) the problem has been reduced to the case that the rotation of the staggered magnetization is restricted to a certain fixed plane. This simplification yields a representative and realistic approximative model replacing the general model (8). It is amenable to a complete analysis of the possible solutions for magnetic structures. The boundaries of their existence in parameter space could be calculated in all detail and a clear physical picture of the formation and evolution of these magnetic states is achieved. Due to the unique combination of those interactions inducing weak ferromagnetism and those stabilizing modulated chiral states, a rich variety of new modulated and localized structures was found to exist in this class of magnetic crystals. In these inhomogeneous states chiral rotation of the staggered magnetization is always accompanied by oscillations of a weak magnetization component in the basal plane (Fig. 1). The modulated states in these systems can be realized as structures with the propagation vector along certain in-plane directions (spirals). We remark that another type of solutions, two-dimensional modulated phases, so-called vortex lattices may also exist. In noncentrosymmtric ferromagnets they are thermodynamically stable mnder applied fields in certain region of the phase space 15 In Ref. 30 vortexlattices in antiferromagnets lacking inversion symmetry with $d=0$, have been studied theoretically. Nucleation of such vortex lattices during the transition from the spinflop phase is discussed in Ref. 37. However, it is still unknown whether these vortex lattices can be thermodynamically stable in antiferromagnetic materials.

We have described one-dimensional localized structures (domain walls or kinks) separating domains of homogeneous states. These differ from similar objects found in many other classes of magnetic materials by oscillations of the local net magnetization and the dependence of their energy on the sense of rotation for the staggered magnetization within the wall. These peculiarities of their properties should be accessible to experimen- tal verification. Furthermore, two-dimensional localized structures with finite sizes (as axisymmetric vortices in the antiferromagnetic phase) are possible topological defects in these systems. They are stabilized only due to the chiral interactions.

In this paper we have deliberately avoided a detailed investigations of the full model (8). Instead, by introducing a simplified model, we have described the general features of the magnetic properties in noncentrosymmetric tetragonal antiferromagnets. We expect that this phenomenological description will provide a guide for further detailed experimental investigation of the known noncentrosymmetric tetragonal antiferromagnets and for a search of new crystals belonging to this group.

We also briefly indicate here possible further directions of the theoretical investigations. Fourth-order anisotropies are needed to describe orientational processes in the basal plane and the peculiarities of magnetic properties near the spin-flop field. Future theoretical investigation also should include the stray field effects responsible for multidomain states near the firstorder phase transitions. Similar investigations within the general model (8) pose a much more complex and challenging task. This functional can be considered as a generalized version of the nonlinear $\sigma$-model, one of the basic models in the theory of nonlinear physics and solitons. It is related to many other models in condensed matter physics. 42.43 .44 .45 .49 The further development of the theory should involve the investigation of vortices and vortex lattices as done for other noncentrosymmetric models.15.30 Similar multidimensional localized solutions of nonlinear field equations are iptengely studied in many other fields of modern physics. 42.44 . 20 Nucleation and evolution of such one-dimensional and two dimensional modulated patterns have deep physical relations to similar patterns in superconductivity,57 liquid crystals, 49 and other condensed matter systems and even in modern cosmological models. 8

\section{Acknowledgments}

We thank M.D. Lumsden and J.R. Thompson for explanations and communication of unpublished results. A.N.B. thanks H. Eschrig for support and kind hospitality during his stay at IFW Dresden. He acknowledges further support by DFG through Sonderforschungsbereich 422. U.K.R. gratefully acknowledges support by DFG through grant MU 1015/7-1.

\footnotetext{
* Permanent address: Donetsk Institute for Physics and Technology, 340114 Donetsk, Ukraine; ; Electronic address: bogdanov@kinetic.ac.donetsk.ua

$\dagger$ Corresponding author: IFW Dresden, Postfach 270116, D01171 Dresden, Germany. Tel.: +49-351-4659-542; Fax:

+49-351-4659-537; Electronic address: u.roessler@ifwdresden.de

I. E. Dzyaloshinskii, Sov. Phys. JETP 5, 1259 (1957).

2 T. Moriya, Phys. Rev. 120, 91 (1960).

3 L. Néel, Rev. Mod. Phys. 25, 58 (1953); A. S. Borovik-
} 
Romanov and M. P. Orlova, Soviet Physics JETP- USSR, 4, 531 (1957).

4 S. Chikazumi, Physics of Ferromagnetism, Oxford, Clarendon Press, 1997.

${ }^{5}$ L. M. Sandratskii, Adv. Phys. 47, 91 (1998).

${ }^{6}$ H. M. Luo, Y. Y. Hsu, B. N. Lin, Y. P. Chi, T. J. Lee, and H. C. Ku, Phys. Rev. B 60, 13119 (1999); P. L. Gammel, B. Barber, D. Lopez, A. P. Ramirez, D. J. Bishop,S. L. Bud'ko, and P. C. Canfield, Phys. Rev. Lett. 84, 2497 (2000).

7 I. E. Dzyaloshinskii, Sov. Phys. JETP 19, 960 (1964); 20, 223 (1965); 20, 665 (1965).

8 L. D. Landau, E. M. Lifshitz, Statistical Physics. Course of Theoretical Physics, Vol. V, Pergamon, Oxford, 1997.

9 Y. Isikawa, K. Tajima, D. Bloch, and M. Roth, Solid State Comm. 19, 525 (1976); C. Wilkinson, F. Sinclair, and J. B. Forsyth, 5th Conf. on Solid Compounds of Transition Elements. Extended Abstracts, Uppsala 1976, p. 158.

10 P. Bak and M. H. Jensen, J. Phys.C: Solid State Phys. 13, L881 (1980); O. Nakanishi, A. Yanase, A. Hasegawa, and M. Kataoka, Solid State Comm. 35, 995 (1980).

11 Yu. A. Izyumov, Sov. Phys. Usp. 27, 845 (1984).

12 B. Lebech, J. Bernhard, and T. Freltoft, J. Phys.: Condens. Matter 1, 6105 (1989).

${ }^{13}$ K. Adachi, N. Achiwa, and M. Mekata, J. Phys. Soc. Japan 49, 545 (1980); T. Ohyama and A. E. Jacobs, Phys. Rev. B 52, 4389 (1995); S. Maruyama, H. Tanaka, Y. Narumi, K. Kindo, H. Nojiri, M. Motokawa, and K. Nagata, J. Phys. Soc. Japan 70, 859 (2001).

14 W. C. Koehler, J. Appl. Phys. 36, 1078 (1965).

15 A.N. Bogdanov and D.A. Yablonsky, Sov. Phys. JETP 68, 101 (1989).

16 A. Zheludev, G. Shirane, Y. Sasago, N. Koide, and K. Uchinokura, Phys. Rev. B 54, 15163 (1996); see also 55, 11879 (1997).

17 A. Zheludev, S. Maslov, G. Shirane, Y. Sasago, N. Koide, and K. Uchinokura, Phys. Rev. Lett. 78, 4857 (1997); A. Zheludev, S. Maslov, G. Shirane, Y. Sasago, N. Koide, K. Uchinokura, D. A. Tennant, and S. E. Nagler, Phys. Rev. B 56,14006 (1997); A. Zheludev, S. Maslov, G. Shirane, Y. Sasago, N. Koide, and K. Uchinokura, Phys. Rev. B 57, 2968(1998); A. Zheludev, S. Maslov, G. Shirane, I. Tsukuda, T. Masuda, K. Uchinorkura, I. Zaliznyak, R. Erwin, and L. P. Regnault, Phys. Rev. B 59, 11432 (1999).

18 M.D. Lumsden, B.C. Sales, D. Mandrus, S.E. Nagler, and J.R. Thompson, Phys. Rev. Lett. 86, 159 (2001).

19 V. G. Bar'yakhtar, A. N. Bogdanov, and D. A. Yablonskii, Sov. Phys. Usp. 31, 810 (1988).

${ }^{20}$ I. J. Lowe and D. W. Whitson, Phys. Rev. B 6, 3262 (1972); J. W. Lynn, P. Heller, and N. A. Lurie, Phys. Rev. B 16, 5032 (1977).

21 Y. Shapira and S. Foner, Phys. Rev. B 1, 3083 (1970); J. Nogués, L. Morellon, C. Leighton, M. R. Ibarra, and I. K. Schuller, Phys. Rev. B 61, R6455 (2000).

22 Y. Shapira and C. C. Becerra, Phys. Rev. B 16, 4920 (1977); M. Fiebig, D. Fröhlich, and H.-J. Thiele, Phys. Rev. B 54, 12681 (1996); J. S. Dodge, A. B. Schumacher, J.-Y. Bigot, D. S. Chemla, N. Ingle, and M. R. Beasley, Phys. Rev. Lett. 83, 4650 (1999).

23 K. W. Blazey and H. Rohrer, Phys. Rev. 173, 574 (1968); K. W. Blazey, H. Rohrer, and R. Webster Phys. Rev. B 4, 2287 (1971).

24 L. J. DeJongh and A. R. Miedema Adv. Phys. 23, 1 (1974).

${ }^{25}$ K. P. Belov, A. K. Zvezdin, A. M. Kadomtseva, and R. Z.
Levitin, Sov. Phys. Usp., 19, 574 (1976)

26 See, e.g., V. Skumryev, F. Ott, J.M.D. Coey, A. Anane, J.P. Renard, L. Pinsard-Gaudart, and A. Revcolevschi, Eur. Phys. J. B 11, 401 (1999); M. Cestelli Guidi, G. Allodi, R. De Renzi, G. Guidi, M. Hennion, L. Pinsard, and A. Amato, Phys. Rev. B 64, 064414 (2001).

27 Y. Shapira, Phys. Rev. 184, 589 (1969); V. I. Ozhogin and V. G. Shapiro, Sov. Phys. JETP - USSR 28, 915 (1969); Q. A. Pankhurst, C. E. Jonhson, M. F. Thomas J. Phys. C: Solid St. Phys. 19, 7081 (1986); J. Baruchel, Physica B 192,79 (1993).

28 A.N. Bogdanov, Sov. J. Low Temp. Phys. 12, 290 (1986).

29 A.H. Morrish, Canted antiferromagnetism in Hematite, Singapore, World Scientific 1994.

30 A.N. Bogdanov and D.A. Yablonsky, Sov. Phys. JETP 69, 142 (1989); A.N. Bogdanov and A.A. Shestakov, Phys. Sol. State, 40, 1350 (1998).

31 A. Bogdanov and A. Hubert, J. Magn. Magn. Mater. 138, 255 (1994).

32 C. Rebbi, G. Soliani, Solitons and Particles, World Scientific, Singapore, 1984; R. Rajaraman, Solitons and Instantons, North-Holland, Amsterdam, 1982.

33 A. Hubert, R. Schäfer, Magnetic Domains. The analysis of Magnetic Microstructures, Berlin, Springer-Verlag, 1998.

34 A. N. Bogdanov and A. A. Shestakov, Low Temp. Phys. 25, 76 (1999).

35 C. A. Condat, R. A. Guyer, and M. D. Miller, Phys. Rev. B 27, 474 (1985); R. Pandit, C. Tannous, and J. A. Krumhansl, Phys. Rev. B 28, 289 (1983).

36 G.M. Korn and T.M. Korn, Mathematical Handbook for Scientists and Engineers, McGraw-Hill, New York 1968.

37 J. Chovan, N. Papanicolaou, and S. Komineas, Phys. Rev. B 65, 064433 (2002).

38 A. Hubert, Theorie der Domänenwände in geordneten Medien, Springer, Berlin 1974.

39 W. J. Ince and A. Platzker, Phys. Rev. 175, 650 (1968); A. N. Bogdanov, V. T. Telepa, P. P. Shatsky, and D. A. Yablonsky, Sov. Phys. JETP 90, 1738 (1986); A. Scholl, J. Stöhr, J. Luning, J. W. Seo, J. Fompeyrine, H. Siegwart, J. P. Locquet, F. Nolting, S. Anders, E. E. Fullerton, M. R. Scheinfein, and H. A. Padmore, Science 287, 1014 (2000); W. Zhu, L. Seve, R. Sears, B. Sinkovic, and S. S. P. Parkin, Phys. Rev. Lett. 86, 5389 (2001).

40 A. Bogdanov and A. Hubert, phys. stat. sol. (b) 186, 527 (1994); A. Bogdanov, JETP Lett. 62, 247 (1995); A. Bogdanov and A. Hubert, J. Magn. Magn. Mater. 195, 182 (1999).

41 A.N. Bogdanov and A.A. Shestakov, JETP 86, 911 (1998).

42 M. M. Salomaa and G. E. Volovik, Rev. Mod. Phys. 59, 533 (1987); H. Yabu and H. Kuratsuji, J. Phys. A: Math. Gen. 32, 7367 (1999).

43 S. L. Sondhi, A. Karlhede, S. A. Kivelson, and E. H. Rezayi, Phys. Rev. B 47, 16419 (1993); S.V. Iordanskii, Physics - Uspekhi 41, 134 (1998).

44 A.N. Bogdanov and U.K. Rößler, Phys. Rev. Lett. 87, 037203 (2001).

45 V. Pasquier Phys. Lett. B 513,241 (2001).

46 A.A. Belavin and A.M. Polyakov, JETP Lett. 22, 245 (1975).

47 N. D. Mermin, Rev. Mod. Phys. 51, 591 (1979); P. G. de Gennes, J. Prost, The Physics of Liquid Crystals, Clarendon, Oxford 1993.

48 V. L. Berezinskii, Sov. Phys. JETP 32, 493 (1971); J. M. Kosterlitz and D. J. Thouless, J. Phys. C 6, 1181 (1973); 
J. M. Kosterlitz, J. Phys. C 7, 1046 (1974).

49 P. Oswald, J. Baudry, and S. Pirkl, Phys. Rep. 337, 67 (2000).

50 M. D. Lumsden and J. R. Thompson, private communication.

${ }^{51}$ G. Liu and J. E. Greedan J. Sol. State Chem. 114, 499 (1995).

52 G. Petrakovskii D. Velikanov, A. Vorotilov, A. Balaev, K. Sablina, A. Amato, B. Roessli, J. Schefer, and U. Staub, J. Magn. Magn. Mat. 205, 105 (1999).

53 B. Roessli, J. Schefer, G. A. Petrakovskii, B. Ouladdiaf, M. Boehm, U. Staub, A. Vorotilov, and L. Bezmaternikh, Phys. Rev. Lett. 86, 1885 (2001).
54 G. A. Petrakovskii, M.A. Popov, B. Roessli, and B. Ouladdiaf, JETP 93, 809 (2001).

${ }^{55}$ I. Sosnowska, T. Peterlin-Neumaier, and E. Steichele, J. Phys. C 15, 4835 (1982); I. Sosnowska and A.K. Zvezdin, J. Magn. Magn. Mater. 140-144, 167 (1995); A. V. Zalessky, A. A. Frolov, T. A. Khimich, A. A. Bush, V. S. Pokatilov, and A. K. Zvezdin, Europhys. Lett. 50, 547 (2000).

56 J. C. Woolley, A.-M. Lamarche, G. Lamarche, M. Quintero, I. P. Swainson, and T. M. Holden, J. Magn. Magn. Mater. 162, 347 (1996).

57 A. A. Abrikosov, Sov. Phys. JETP 5, 1174 (1957).

58 W. H. Zurek, Phys. Rep. 276, 177 (1996). 